\title{
Search for Multi-flare Neutrino Emissions in 10 yr of IceCube Data from a Catalog of Sources
}

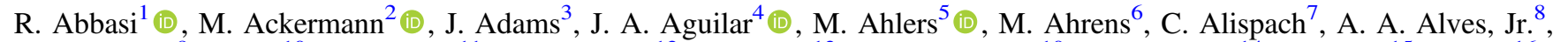

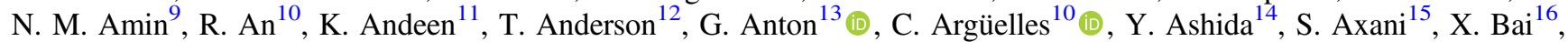

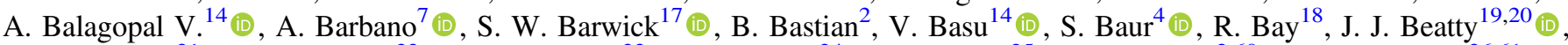

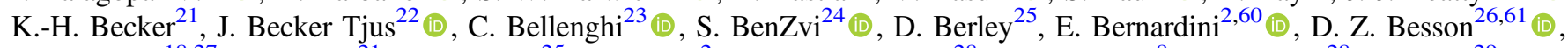

G. Binder ${ }^{18,27}$, D. Bindig ${ }^{21}$, E. Blaufuss ${ }^{25}$ (1) S. Blot ${ }^{2}$ (1) M. Boddenberg ${ }^{28}$, F. Bontempo ${ }^{8}$, J. Borowka ${ }^{28}$, S. Böser ${ }^{29}$ (1),

O. Botner ${ }^{30}$ (1) J. Böttcher ${ }^{28}$, E. Bourbeau ${ }^{5}$, F. Bradascio ${ }^{2}$,, J. Braun $^{14}$, S. Bron ${ }^{7}$, J. Brostean-Kaiser ${ }^{2}$, S. Browne ${ }^{31}$,

A. Burgman ${ }^{30}$ (1) R. T. Burley ${ }^{32}$, R. S. Busse ${ }^{33}$, M. A. Campana ${ }^{34}$ (ㄴ) E. G. Carnie-Bronca ${ }^{32}$, C. Chen ${ }^{35}$ (1) D. D. Chirkin ${ }^{14}$ (1),

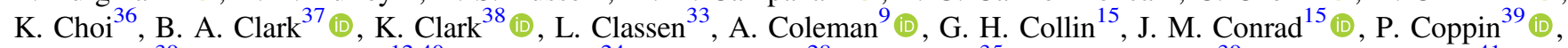

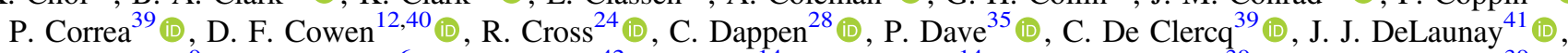

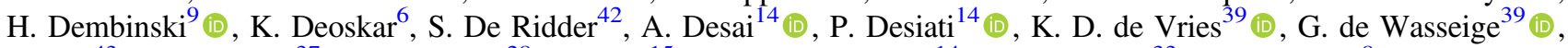
M. de With ${ }^{43}$, T. DeYoung ${ }^{37}$ (1) S. Dharani ${ }^{28}$, A. Diaz ${ }^{15}$ () J. C. Díaz-Vélez ${ }^{14}$ (1) M. Dittmer $^{33}$, H. Dujmovic ${ }^{8}$ (i), M. Dunkman ${ }^{12}$, M. A. DuVernois ${ }^{14}$ (1), E. Dvorak ${ }^{16}$, T. Ehrhardt ${ }^{29}$, P. Eller $^{23}$ (1) R. Engel ${ }^{8,31}$, H. Erpenbeck $^{28}$, J. Evans ${ }^{25}$, P. A. Evenson ${ }^{9}$ (i),

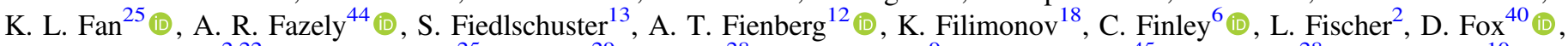
A. Franckowiak ${ }^{2,22}$ (1), E. Friedman ${ }^{25}$, A. Fritz ${ }^{29}$, P. .ürst $^{28}$, T. K. Gaisser ${ }^{9}$ (i), J. Gallagher ${ }^{45}$, E. Ganster ${ }^{28}$ (1) A. Aarcia ${ }^{10}$ (i),

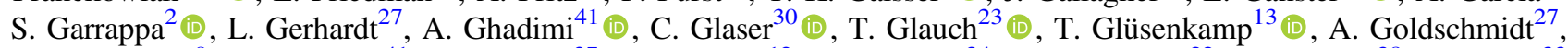
J. G. Gonzalez ${ }^{9}$, S. Goswami ${ }^{41}$ (1), D. Grant ${ }^{37}$, T. Grégoire ${ }^{12}$, S. Griswold ${ }^{24}$ (1) M. Gündüz ${ }^{22}$, C. Günther ${ }^{28}$, C. Haack ${ }^{23}$,

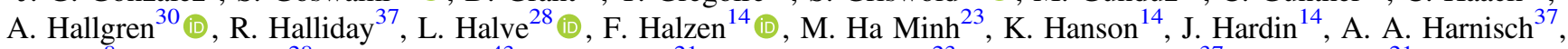
A. Haungs ${ }^{8}$ (D) S. Hauser ${ }^{28}$, D. Hebecker ${ }^{43}$, K. Helbing ${ }^{21}$ (1) , F. Henningsen ${ }^{23}$ (i), E. C. Hettinger ${ }^{37}$, S. Hickford ${ }^{21}$, J. Hignight ${ }^{46}$, C. Hill ${ }^{47}$ (1) G. C. Hill ${ }^{32}$, K. D. Hoffman ${ }^{25}$, R. Hoffmann ${ }^{21}$, T. Hoinka ${ }^{48}$, B. Hokanson-Fasig ${ }^{14}$, K. Hoshina ${ }^{14,62}$, F. Huang ${ }^{12}$ (1),

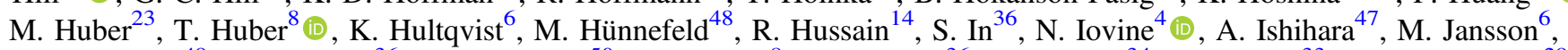

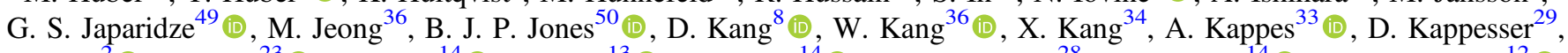

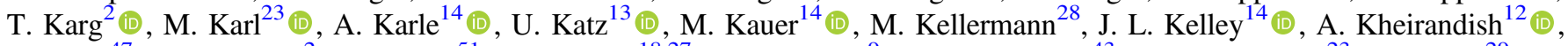
K. Kin ${ }^{47}$, T. Kintscher ${ }^{2}$, J. Kiryluk ${ }^{51}$, S. R. Klein ${ }^{18,27}$ (1) , R. Koirala ${ }^{9}$ (1), H. Kolanoski ${ }^{43}$ (1) , T. Kontrimas ${ }^{23}$, L. Köpke ${ }^{29}$ (1),

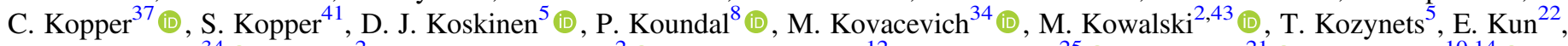

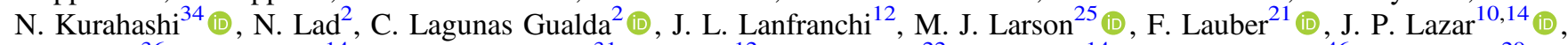

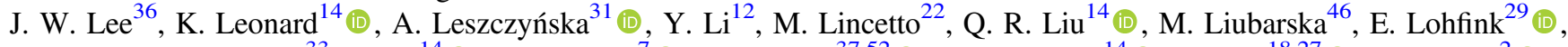

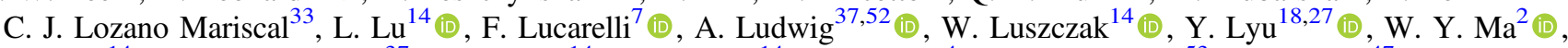

J. Madsen ${ }^{14}\left(\mathbb{D}\right.$, K. B. M. Mahn ${ }^{37}$, Y. Makino ${ }^{14}$, S. Mancina ${ }^{14}$, I. C. Mariş ${ }^{4}\left(\mathbb{D}\right.$, R. Maruyama ${ }^{53}$ (D), K. Mase ${ }^{47}$, T. McElroy $^{46}$, F. McNally ${ }^{54}$ (1) J. V. Mead ${ }^{5}$, K. Meagher ${ }^{14}$ (D) A. Medina ${ }^{20}$, M. Meier ${ }^{47}$ (D), S. Meighen-Berger ${ }^{23}$ (D) , J. Micallef ${ }^{37}$, D. Mockler ${ }^{4}$,

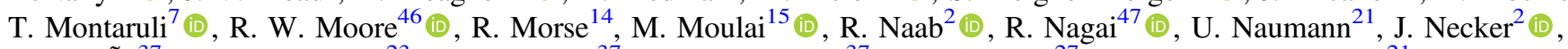

L. V. Nguyễn ${ }^{37}$, H. Niederhausen ${ }^{23}$ (1) M. U. Nisa ${ }^{37}$ (D) S. C. Nowicki ${ }^{37}$, D. R. Nygren ${ }^{27}$, A. Obertacke Pollmann ${ }^{21}$ (1) , M. Oehler ${ }^{8}$, B. Oeyen ${ }^{42}$ (1) A. Olivas $^{25}$, E. O'Sullivan ${ }^{30}$ (D) H. Pandya ${ }^{9}$ (i), D. V. Pankova ${ }^{12}$, N. Park ${ }^{38}$ (1), G. K. Parker ${ }^{50}$, E. N. Paudel ${ }^{9}$ (i),

L. Paul ${ }^{11}$, C. Pérez de los $\operatorname{Heros}^{30}{ }^{(1)}$, L. Peters ${ }^{28}$, J. Peterson ${ }^{14}$ (1), S. Philippen ${ }^{28}$, D. Pieloth ${ }^{48}$, S. Pieper ${ }^{21}$, M. Pittermann ${ }^{31}$, A. Pizzuto $^{14}$ (i), M. Plum ${ }^{11}$ (1), Y. Popovych ${ }^{29}$, A. Porcelli ${ }^{42}$ (1), M. Prado Rodriguez ${ }^{14}$, P. B. Price ${ }^{18}$, B. Pries ${ }^{37}$, G. T. Przybylski $^{27}$, C. Raab $^{4}(1)$, A. Raissi ${ }^{3}$, M. Rameez ${ }^{5}$ (1) K. Rawlins ${ }^{55}$, I. C. Rea ${ }^{23}$, A. Rehman ${ }^{9}$ (D) P. Reichherzer ${ }^{22}$, R. Reimann ${ }^{28}$ (D), G. Renzi ${ }^{4}$, E. Resconi ${ }^{23}$ (1) , S. Reusch ${ }^{2}$ (1) , W. Rhode ${ }^{48}$ (1) , M. Richman ${ }^{34}$, B. Riedel ${ }^{14}$ (1) , E. J. Roberts ${ }^{32}$, S. Robertson ${ }^{18,27}$, G. Roellinghoff ${ }^{36}$, M. Rongen ${ }^{29}$ (1) , C. Rott $^{36,56}$ (i) T. Ruhe ${ }^{48}$, D. Ryckbosch ${ }^{42}$ (i), D. Rysewyk Cantu ${ }^{37}$ (), I. Safa ${ }^{10,14}$ (i), J. Saffer ${ }^{31}$,

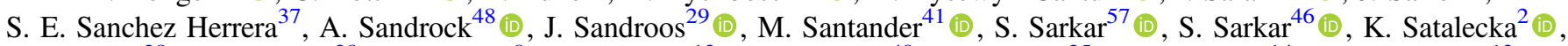

M. Scharf ${ }^{28}$, M. Schaufel ${ }^{28}$, H. Schieler ${ }^{8}$, S. Schindler ${ }^{13}$, P. Schlunder ${ }^{48}$, T. Schmidt ${ }^{25}$, A. Schneider ${ }^{14}$ (D) J. Schneider ${ }^{13}$ (D),

F. G. Schröder ${ }^{8,9}$ (1) , L. Schumacher ${ }^{23}$ (1) , G. Schwefer ${ }^{28}$, S. Sclafani ${ }^{34}$ (1), D. Seckel ${ }^{9}$, S. Seunarine ${ }^{58}$, A. Sharma ${ }^{30}$ (i), S. Shefali ${ }^{31}$,

M. Silva ${ }^{14}$ (i) , B. Skrzypek ${ }^{10}$, B. Smithers ${ }^{50}$ (i) , R. Snihur ${ }^{14}$, J. Soedingrekso ${ }^{48}$ (i), D. Soldin ${ }^{9}$ (i), C. Spannfellner ${ }^{23}$,

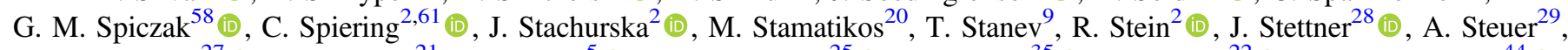

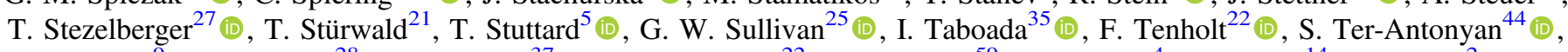

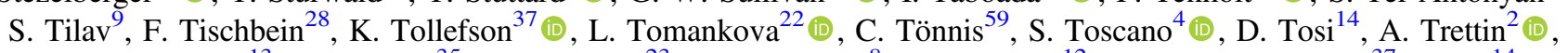

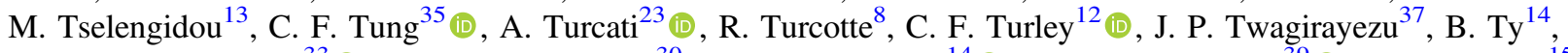

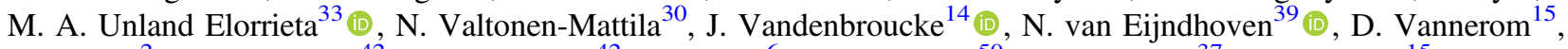

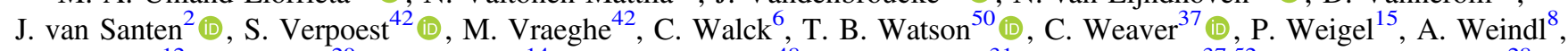

M. J. Weiss ${ }^{12}$, J. Weldert ${ }^{29}$ (1), C. Wendt ${ }^{14}$ (1) J. Werthebach ${ }^{48}$, M. Weyrauch ${ }^{31}$, N. Whitehorn ${ }^{37,52}$ (D), C. H. Wiebusch ${ }^{28}$ (1), D. R. Williams ${ }^{41}$, M. Wolf ${ }^{23}$ (1), K. Woschnagg ${ }^{18}$, G. Wrede ${ }^{13}$, J. Wulff ${ }^{22}$, X. W. Xu ${ }^{44}$, Y. Xu ${ }^{51}$, J. P. Yanez ${ }^{46}$, S. Yoshida $^{47}$ (D), S. Yu ${ }^{37}$, T. Yuan ${ }^{14}$ (1), and Z. Zhang ${ }^{51}$ (1) IceCube Collaboration 


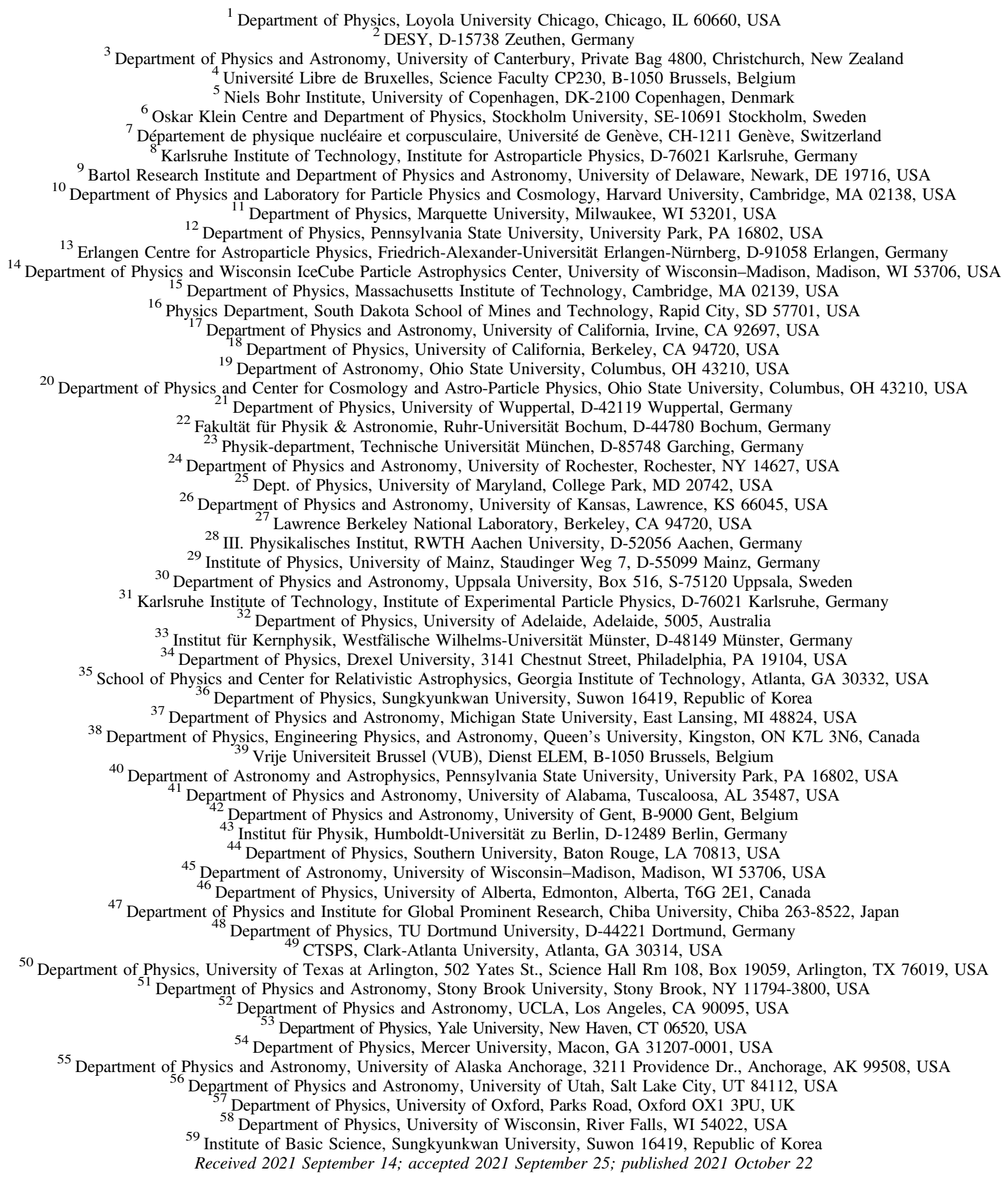

\begin{abstract}
A recent time-integrated analysis of a catalog of 110 candidate neutrino sources revealed a cumulative neutrino excess in the data collected by IceCube between 2008 April 6 and 2018 July 10. This excess, inconsistent with the background hypothesis in the Northern Hemisphere at the 3.3 $\sigma$ level, is associated with four sources: NGC 1068, TXS 0506+056, PKS 1424+240, and GB6 J1542+6129. This Letter presents two time-dependent neutrino emission searches on the same data sample and catalog: a point-source search that looks for the most significant
\end{abstract}

\footnotetext{
60 also at Università di Padova, I-35131 Padova, Italy.

61 also at National Research Nuclear University, Moscow Engineering Physics Institute (MEPhI), Moscow 115409, Russia.

62 also at Earthquake Research Institute, University of Tokyo, Bunkyo, Tokyo 113-0032, Japan.
} 
time-dependent source of the catalog by combining space, energy, and time information of the events, and a population test based on binomial statistics that looks for a cumulative time-dependent neutrino excess from a subset of sources. Compared to previous time-dependent searches, these analyses enable a feature to possibly find multiple flares from a single direction with an unbinned maximum-likelihood method. M87 is found to be the most significant time-dependent source of this catalog at the level of $1.7 \sigma$ post-trial, and TXS $0506+056$ is the only source for which two flares are reconstructed. The binomial test reports a cumulative time-dependent neutrino excess in the Northern Hemisphere at the level of 3.0 $\sigma$ associated with four sources: M87, TXS 0506+056, GB6 $\mathrm{J} 1542+6129$, and NGC 1068 .

Unified Astronomy Thesaurus concepts: Neutrino astronomy (1100); High energy astrophysics (739); Particle astrophysics (96)

Supporting material: machine-readable table

\section{Introduction}

After more than $100 \mathrm{yr}$ since their discovery, the origin and acceleration processes of cosmic rays (CRs) remain unsolved. Relevant hints exist, one being provided by a neutrino event detected by IceCube with a most probable energy of $290 \mathrm{TeV}$ which triggered follow-up gamma-ray observations (Aartsen et al. 2018a). These observations identified in the $50 \%$ containment region for the arrival direction of the IceCube event a classified BL Lac object, though possibly a flat-spectrum radio quasar (FSRQ, Padovani et al. 2019), at redshift $z=0.34$, known as TXS $0506+056$. It was in a flaring state (Aartsen et al. 2018a) with a chance correlation between the neutrino event and the photon counterpart rejected at the $3 \sigma$ level. The intriguing aspect of the possible coincidence between the neutrino event and the gammaray flare hints at TXS $0506+056$ being a potential CR source. Additionally, in the analysis of the data prior to the event alert IceCube found a neutrino flare of 110 day duration between 2014/2015 (Aartsen et al. 2018b) at a significance of $3.7 \sigma$ if a Gaussian time window is assumed. In this case, no clear flare has been identified in available gamma-ray data from TXS 0506+056 (Garrappa et al. 2019; Padovani et al. 2018). The total contribution of the observed TXS 0506+056 neutrino flares to the diffuse astrophysical flux observed by IceCube (Aartsen et al. 2013a, 2014a, 2016, 2017c) is at most a few percent (Aartsen et al. 2018b). In addition, time-integrated upper limits on stacked catalogs of classes of sources (e.g., tidal disruption events [Stein 2020], blazars [Aartsen et al. 2017a], gamma-ray bursts [Aartsen et al. 2017d], compact binary mergers [Aartsen et al. 2020a], and pulsar wind nebulae [Aartsen et al. 2020b]), constrain their contribution to the measured diffuse flux. While these limits depend on assumptions on the emission of such classes of sources, such as their spectral shapes and their uniformity within the class, they indicate that there might be a mixture of contributing classes and still unidentified contributors.

Recently, IceCube performed another analysis on neutrino sources: a time-integrated search for point-like neutrino source signals using 10 years of data (Aartsen et al. 2020c). This search used a maximum-likelihood (ML) method to test the locations of a catalog of 110 selected sources and the full sky. As an intriguing coincidence, the two searches found the hottest spot to be a region including the Seyfert II galaxy NGC 1068, with a significance reported from the catalog search of $2.9 \sigma$. Additionally, a population study of the catalog revealed a $3.3 \sigma$ level incompatibility of the neutrino events from the directions of four northern sources with respect to the estimated background: NGC 1068, TXS 0506+056, PKS 1424+240, and GB6 J1542+6129.

To fully investigate this catalog of sources, this Letter shows the results of a complementary time-dependent study. Time- dependent searches are particularly interesting not only because of their better sensitivity to time-integrated searches for flares of duration $\lesssim 200$ days due to the suppression of the timeconstant background of atmospheric neutrinos, but also because flare events are particularly suitable periods for neutrino production in blazars. In fact, the injection rate of accelerated protons and the density of target photon fields for photomeson interactions can be noticeably increased during flaring periods of blazars, leading to an enhanced neutrino luminosity $L_{\nu} \propto L_{\gamma}^{1.5-2}$ (see Zhang et al. 2020 and references therein), where $L_{\gamma}$ is the photon luminosity. Apart from the aforementioned evidence of the 2014/2015 flare from the direction of TXS $0506+056$, other IceCube time-dependent searches did not find any significant excess. Nevertheless, they constrained specific emission models (Abbasi et al. 2021a) or set upper limits on the neutrino emission from selected sources (Aartsen et al. 2015). Triggered searches adopt lightcurves or flare directions from gamma-ray experiments, while sky scans search for the largest flares anywhere in the sky. In this paper, we extend these searches to a multiple flare scan based on an ML method.

\section{Apparatus and Data Sample}

The IceCube Neutrino Observatory (Achterberg et al. 2006) is a $1 \mathrm{~km}^{3}$ sized neutrino telescope optimized for detection of highenergy neutrinos above $\sim 100 \mathrm{GeV}$. It is located at the South Pole at a depth from $\sim 1.5$ to $2.5 \mathrm{~km}$ in the Antarctic ice. It consists of 86 strings instrumented with 5160 digital optical modules, each equipped with a 10 inch photomultiplier tube (Abbasi et al. 2010) in a pressure-resistant sphere with associated digitizing electronics (Abbasi et al. 2009).

The data set used in this analysis comprises $10 \mathrm{yr}$ of IceCube data from 2008 April 6 to 2018 July 10 (Aartsen et al. 2020c; Abbasi et al. 2021b). It includes data from partially built detector configurations, with 40, 59, and 79 strings (IC40, IC59, and IC79) described in Abbasi et al. (2011), Aartsen et al. (2013b, 2017b), and from the full detector configuration of 86 strings (IC86-I), described in Aartsen et al. (2014b). An updated selection of tracklike events that reduces the atmospheric background described in Aartsen et al. (2020c) is applied to the data recorded in 2012-2018. It leads to an all-sky event rate of $\sim 4 \mathrm{mHz}$, dominated by muons from interactions of atmospheric neutrinos from the Northern Hemisphere (up-going region, decl. $\delta \geqslant-5^{\circ}$ ) and by high-energy, well-reconstructed atmospheric muons from the Southern Hemisphere (downgoing region, $\delta<-5^{\circ}$ ). The resulting median angular resolution (the difference between the reconstructed simulated event direction and the true primary neutrino) is smaller than 0.60 above $10 \mathrm{TeV}$, where it shows an improvement of 


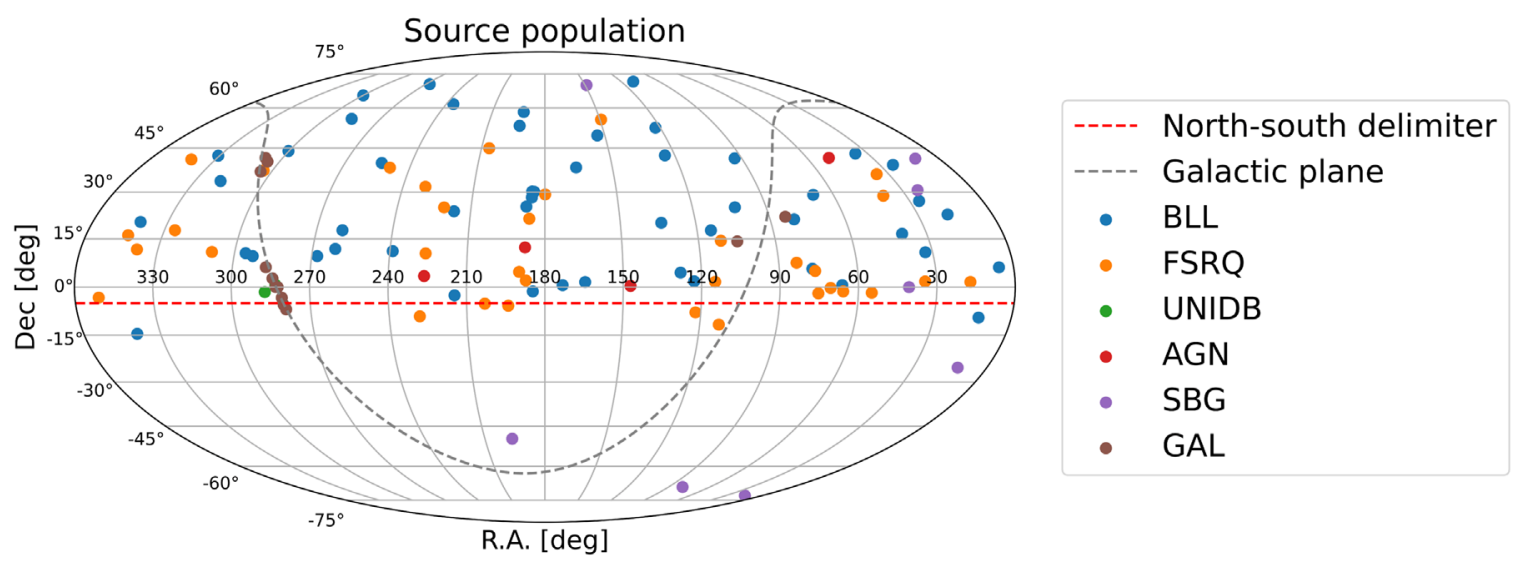

Figure 1. Distribution of the sources in the catalog in equatorial coordinates. They are classified as BL Lacs (BLLs), flat-spectrum radio quasars (FSRQs), active galactic nuclei (AGNs), starburst galaxies (SBGs), unidentified blazars (UNIDBs), and Galactic sources (GALs). The red line divides the Northern Hemisphere (upgoing region) and the Southern Hemisphere (downgoing region) at decl. $\delta=-5^{\circ}$, where the background is substantially different.

$\sim 10 \%$ with respect to the previous event selection (Aartsen et al. 2017b).

The selection of the candidate neutrino sources used in these analyses is described in Aartsen et al. (2020c). The catalog is composed of extragalactic sources selected from the FermiLAT 4FGL catalog (Abdollahi et al. 2020) that include eight starburst galaxies detected by Fermi-LAT, which may host hadronic interactions of CRs with ambient matter and galactic sources from TeVCat (Wakely \& Horan 2008) and gammaCat (gammaCat., 2018). The catalog is aimed at maximizing the detection probability of IceCube, while considering relevant $\mathrm{GeV}-\mathrm{TeV}$ information from gamma-ray experiments. In total, 110 sources are selected (see Figure 1), 97 located in the Northern Hemisphere and 13 in the Southern one.

\section{Data Analysis Methods}

The presented analyses are based on an unbinned ML method similar to previous IceCube analyses, extended to allow the detection of multiple flares and to handle different IceCube samples (IC40, IC59, IC79, IC86-I, IC86-II-VII) with different detector configurations. Since each IceCube sample is independent, the total $10 \mathrm{yr}$ likelihood $\mathcal{L}$ is defined as the product of the likelihoods of each single IceCube sample $\mathcal{L}_{j}$ :

$$
\mathcal{L}\left(\boldsymbol{n}_{s}, \boldsymbol{\gamma}, \boldsymbol{t}_{0}, \boldsymbol{\sigma}_{T}\right)=\prod_{j=\mathrm{sample}} \mathcal{L}_{j}\left(\boldsymbol{n}_{s, j}, \gamma, \boldsymbol{t}_{0}, \boldsymbol{\sigma}_{T}\right),
$$

where $\mathcal{L}_{j}$ is defined as

$$
\begin{aligned}
& \mathcal{L}_{j}\left(\boldsymbol{n}_{s, j}, \boldsymbol{\gamma}, \boldsymbol{t}_{0}, \boldsymbol{\sigma}_{T}\right) \\
& \quad=\prod_{i=1}^{N_{j}}\left[\frac{\sum_{f=\text { flares }} n_{s, j}^{f} \mathcal{S}_{j}\left(\left|\boldsymbol{x}_{s}-\boldsymbol{x}_{i}\right|, \sigma_{i}, E_{i}, t_{i} ; \gamma^{f}, t_{0}^{f}, \sigma_{T}^{f}\right)}{N_{j}}\right. \\
& \left.\quad+\left(1-\frac{\sum_{f} n_{s, j}^{f}}{N_{j}}\right) \mathcal{B}_{j}\left(\sin \delta_{i}, E_{i}\right)\right] .
\end{aligned}
$$

For each flare $f$, the likelihood in Equation (1) is a function of four parameters described below: the total number of signallike events in the flare $n_{s}^{f}$, the flare spectral index $\gamma^{f}$, the flaring time $t_{0}^{f}$, and the flare duration $\sigma_{T}^{f}$. They are denoted in bold in the likelihood arguments to indicate that there are as many sets of these four parameters as the number of flares. For each flare $f, n_{s, j}^{f}$ in Equation (2) denotes the partial contribution of the $j$ th sample to the total number of signal-like events in that flare, such that $n_{s}^{f}=\sum_{j} n_{s, j}^{f}$. Such partial contribution $n_{s, j}^{f}$ is estimated from the relative effective area of the IceCube configuration of the $j$ th sample (determined by Monte Carlo simulations of the detector and varying with spectral index and decl.) and the fraction of time that the fth flare stretches on the data-taking period of the $j$ th sample.

For each IceCube sample $j$, with $N_{j}$ total events, the likelihood in Equation (2) is constructed from a single-flare signal probability density function (PDF) $\mathcal{S}_{j}$, weighted by $n_{s, j}^{f}$ and summed over all flares from a source (multi-flare signal PDF), and a background PDF $\mathcal{B}_{j}$. The single-flare signal PDF and the background PDF are the product of space, energy, and time PDFs, as also described in Aartsen et al. (2015). The spatial signal PDF assumes a cluster of events distributed according to a 2D Gaussian around the source position $\boldsymbol{x}_{\boldsymbol{s}}$, with $\sigma_{i}$ being the estimated angular uncertainty on the $\boldsymbol{x}_{\boldsymbol{i}}$ position of the $i$ th event. For the signal energy PDF, that depends on the decl. $\delta_{i}$ and the energy proxy $E_{i}$ of the events (the energy as measured by IceCube from visible light released in the detector by muon tracks), an unbroken power law $\propto E^{-\gamma^{f}}$ is used. The spectral index $\gamma^{f}$ is bound within $1 \leqslant \gamma^{f} \leqslant 4$ and can be different for each flare $f$. The signal time PDF of each flare $f$ is provided by a onedimensional Gaussian $\propto \exp \left[-\left(t_{i}-t_{0}^{f}\right)^{2} /\left(2 \sigma_{T}^{f 2}\right)\right]$, where $t_{i}$ is the time of the $i$ th event. Its normalization is such that the integral of the time PDF across the up times of each IceCube sample is 1 . The central time of each Gaussian flare $t_{0}^{f}$ is constrained within the $10 \mathrm{yr}$ period of the analyzed data and the flare duration $\sigma_{T}^{f}$ cannot exceed an upper limit of 200 days, above which time-integrated searches are more sensitive than time-dependent ones. For computational efficiency, the signal time PDF of each flare is truncated at $\pm 4 \sigma_{T}^{f}$, where the flare can be considered concluded.

The spatial background PDF is obtained through a datadriven method by scrambling the time of the events and correcting the R.A. accordingly, assuming fixed local coordinates (azimuth, zenith). It depends only on the decl. $\delta_{i}$ of the events and it is uniform in R.A. Due to the natural tendency of the reconstruction to be more efficient if the direction of the source is aligned with the strings of the detector, an azimuthdependent correction is applied to the spatial background PDF. Such correction is relevant for timescales shorter than one day, whereas it is negligible for longer timescales since any azimuth dependency is averaged out by the Earth rotation. The background energy PDF is taken from scrambled data as well 
and it is fully described in Aartsen et al. (2013b). It depends on the decl. $\delta_{i}$ and the energy proxy $E_{i}$ of the events. The background time PDF is uniform, as expected for atmospheric muons and neutrinos if seasonal sinusoidal variations are neglected. The maximal amplitude for these variations is $10 \%$ for the downgoing muons produced in the polar atmosphere and smaller for atmospheric neutrinos coming from all latitudes (Desiati 2013).

The test statistic (TS) is defined as:

$$
\begin{aligned}
\mathrm{TS} & =-2 \ln \left[\frac{1}{2}\left(\prod_{f=\text { flares }} \frac{T_{\text {live }}}{\hat{\sigma}_{T}^{f} I\left[\hat{t}_{0}^{f}, \hat{\sigma}_{T}^{f}\right]}\right)\right. \\
& \left.\times \frac{\mathcal{L}\left(\boldsymbol{n}_{s}=\mathbf{0}\right)}{\mathcal{L}\left(\hat{\boldsymbol{n}}_{s}, \hat{\boldsymbol{\gamma}}, \hat{\boldsymbol{t}}_{0}, \hat{\boldsymbol{\sigma}}_{T}\right)}\right]
\end{aligned}
$$

where the parameters that maximize the likelihood function in Equation (1) are denoted with a hat and $\mathcal{L}\left(\boldsymbol{n}_{s}=\mathbf{0}\right)$ is the background likelihood, obtained from Equation (1) by setting $n_{s}^{f}=0$ for all the flares. The likelihood ratio is multiplied by a marginalization term intended to penalize short flares, similarly used in previous time-dependent single-flare IceCube analyses to correct a natural bias of the likelihood toward selecting short flares. This was discussed in Braun et al. (2010) for the singleflare analysis. For the multi-flare analysis, the numerical factor $1 / 2$ in the equation above is chosen such that the marginalization term has the same form as the single-flare one when the true hypothesis is a single flare. The factor $0<I\left[\hat{t}_{0}^{f}, \hat{\sigma}_{T}^{f}\right]<1$ is defined as

$$
I\left[\hat{t}_{0}^{f}, \hat{\sigma}_{T}^{f}\right]=\int_{T_{\mathrm{ive}}} \frac{1}{\sqrt{2 \pi} \sigma_{T}^{f}} \exp \left[-\frac{\left(t-t_{0}^{f}\right)^{2}}{2 \sigma_{T}^{f 2}}\right] d t,
$$

where $T_{\text {live }}$ is the full period of the analysis. It is introduced in this analysis to correct for boundary effects of Gaussianmodeled time-dependent searches.

A pre-selection of interesting time frames is made by evaluating the TS of the events, looking for clusters of highenergy events in time and space, and imposing that TS $\geqslant 2$. This value is chosen to reduce the reconstruction probability of "fake" multiple flares to $\lesssim 0.1 \%$ under the null hypothesis (see Appendix B). Clusters of events fulfilling the above requirement are called candidate flares. For the candidate flares with central times $t_{0}^{f}$ overlapping within $\pm 4 \sigma_{T}^{f}$, only the one with the highest TS is retained. If no candidate flares are found, the preselection reports as a candidate flare the one cluster with the highest TS. The parameters of these candidate flares are used as seed values for the ML fit.

The first analysis presented in this Letter, the point-source search, looks for the most significant flaring source in each hemisphere. To do so, the TS is maximized at the location of each source and converted into a local pre-trial $p$ value $p_{\text {loc }}$ by comparing the observed result with a distribution of TS produced under the null hypothesis $\left(\boldsymbol{n}_{s}=\mathbf{0}\right)$. The source corresponding to the lowest pre-trial $p$ value in the respective hemisphere is reported, together with its post-trial $p$ value after accounting for the number of trials, i.e., often called the lookelsewhere effect. The post-trial $p$ value is estimated in each hemisphere by repeating the analysis on background scrambles, picking up the lowest $p$ value in each scramble and counting the fraction of such background $p$ values that are smaller than the lowest pre-trial $p$ value observed in the data.

The second analysis presented in this paper, a population study, is a binomial test that looks for an excess of neutrino emission from a subset of sources that are not strong enough to emerge individually. It aims at determining whether the pre-trial $p$ values observed in a particular hemisphere are compatible as a whole with a background scenario. The $p$ values of the sources are ranked from the lowest to the highest and in each hemisphere the pre-trial binomial $p$ value $P_{\text {bin }}(k)$ is calculated as a function of the source index $k$ (O'Sullivan and Finley 2019; Aartsen et al. 2020c):

$$
P_{\text {bin }}(k)=\sum_{m=k}^{N} \frac{N !}{(N-m) ! m !} p_{k}^{m}\left(1-p_{k}\right)^{N-m}
$$

Here $p_{k}$ is the pre-trial $p$ value of the $k$ th source in the sorted list of $N$ sources ( $N=97$ in the North, $N=13$ in the South). After scanning all the source indices $k$, the smallest binomial $p$ value is corrected for trials and reported as post-trial in each hemisphere, together with the corresponding number of sources. The post-trial binomial $p$ value is estimated in each hemisphere by producing many background realizations of the catalog, picking up the smallest binomial $p$ value in each background realization and counting the fraction of such background binomial $p$ values that are smaller than the binomial $p$ value observed in the data.

\section{Results}

The point-source search identifies M87 as the most significant source in the Northern Hemisphere, with a pre-trial $p$ value of $p_{\text {loc }}=4.6 \times 10^{-4}$, which becomes $4.3 \times 10^{-2}(1.7 \sigma)$ post-trial. In the Southern Hemisphere, the most significant source is PKS 2233-148 with a pre-trial $p$ value of $p_{\text {loc }}=0.092$ and post-trial $p$ value of 0.72 . TXS $0506+056$ is the only source of the catalog for which two flares are found. The time profiles of the neutrino flares reconstructed by this analysis at the location of each source, together with their pre-trial significance $\sigma_{\text {loc }}^{f}$, are visualized in Figure 2. For the sake of clarity, the flare significance is denoted as $\sigma_{\mathrm{loc}}^{f}$ while the overall multi-flare significance is referred to as $\sigma_{\mathrm{loc}}=\sqrt{\sum_{f} \sigma_{\mathrm{loc}}^{f 2}}$. For single-flare sources (all but TXS 0506+056) the flare and multi-flare significances coincide.

The cumulative distributions of pre-trial $p$ values at the location of the sources of the catalog, used as inputs to the population study, are shown in Figure 3.

The pre-trial binomial $p$ value is shown in Figure 4 as a function of the source index $k$. The smallest binomial $p$ value is selected in each hemisphere and converted into a post-trial binomial $p$ value as described in Section 3. In the Northern Hemisphere the smallest pre-trial binomial $p$ value is $7.3 \times 10^{-5}(3.8 \sigma)$ when $k=4$ sources are considered (M87, TXS 0506+056, GB6 J1542+6129, NGC 1068), corresponding to a post-trial $p$ value of $1.6 \times 10^{-3}(3.0 \sigma)$. In the Southern hemisphere the smallest pre-trial binomial $p$ value is 0.71 , obtained by $k=1$ source (PKS 2233-148) and corresponding to a post-trial $p$ value of 0.89 .

The results of the two searches are summarized in Table 1. Having not found any significant time-dependent excess, upper limits on the neutrino emission from the sources of the catalog are estimated as discussed in Appendix A, using Equation (A1) and $\mathrm{A} 2$. 


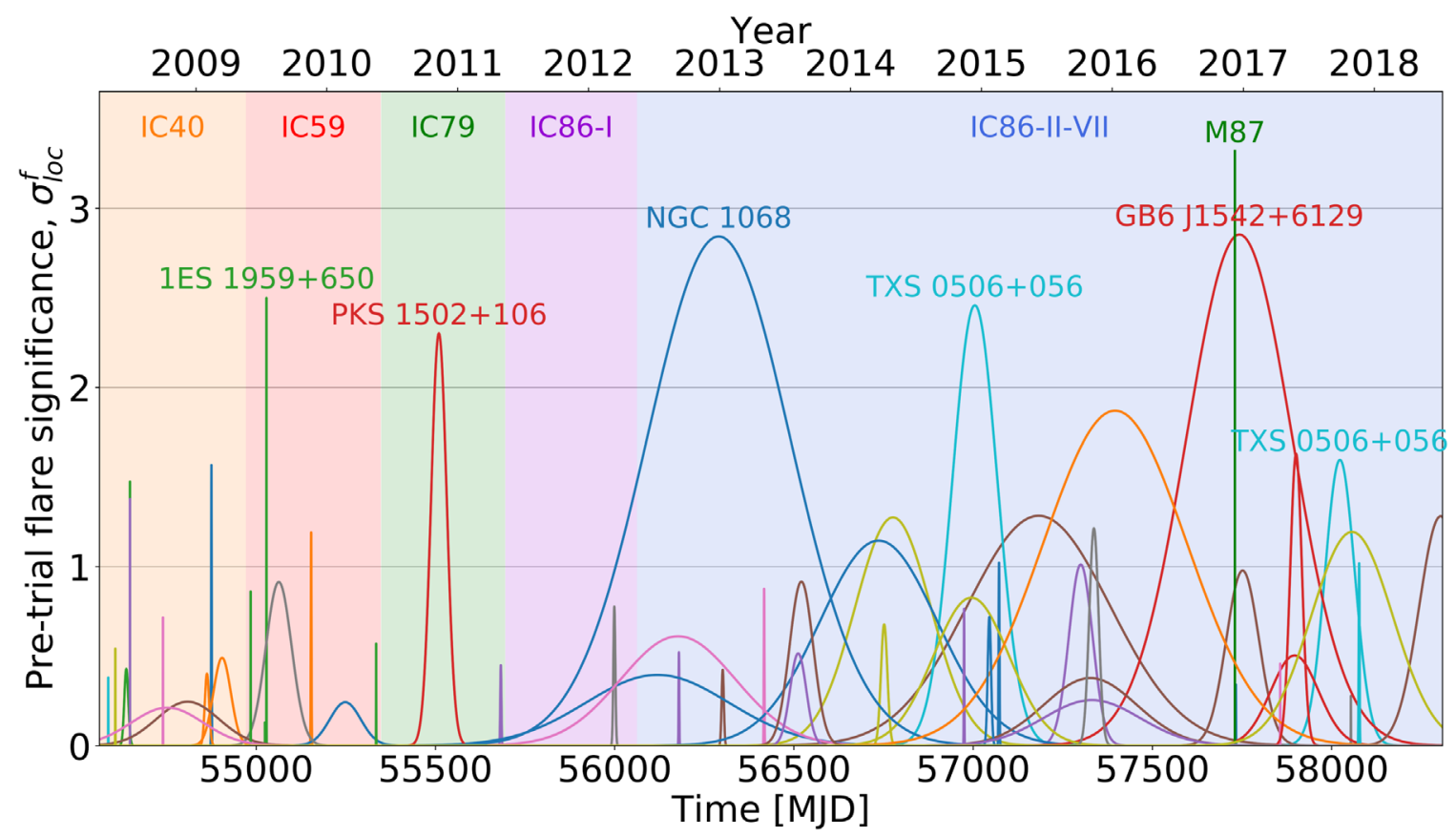

Figure 2. Pre-trial flare significance $\sigma_{\mathrm{loc}}^{f}$ for the sources of the catalog. For all sources, a single flare has been found, except for TXS $0506+056$ for which two flares were found. In this case, the pre-trial significance of each individual flare is calculated as described in Appendix D. The sources of the catalog with multi-flare pre-trial significance $\sigma_{\mathrm{loc}} \geqslant 2$ are labeled with their names.
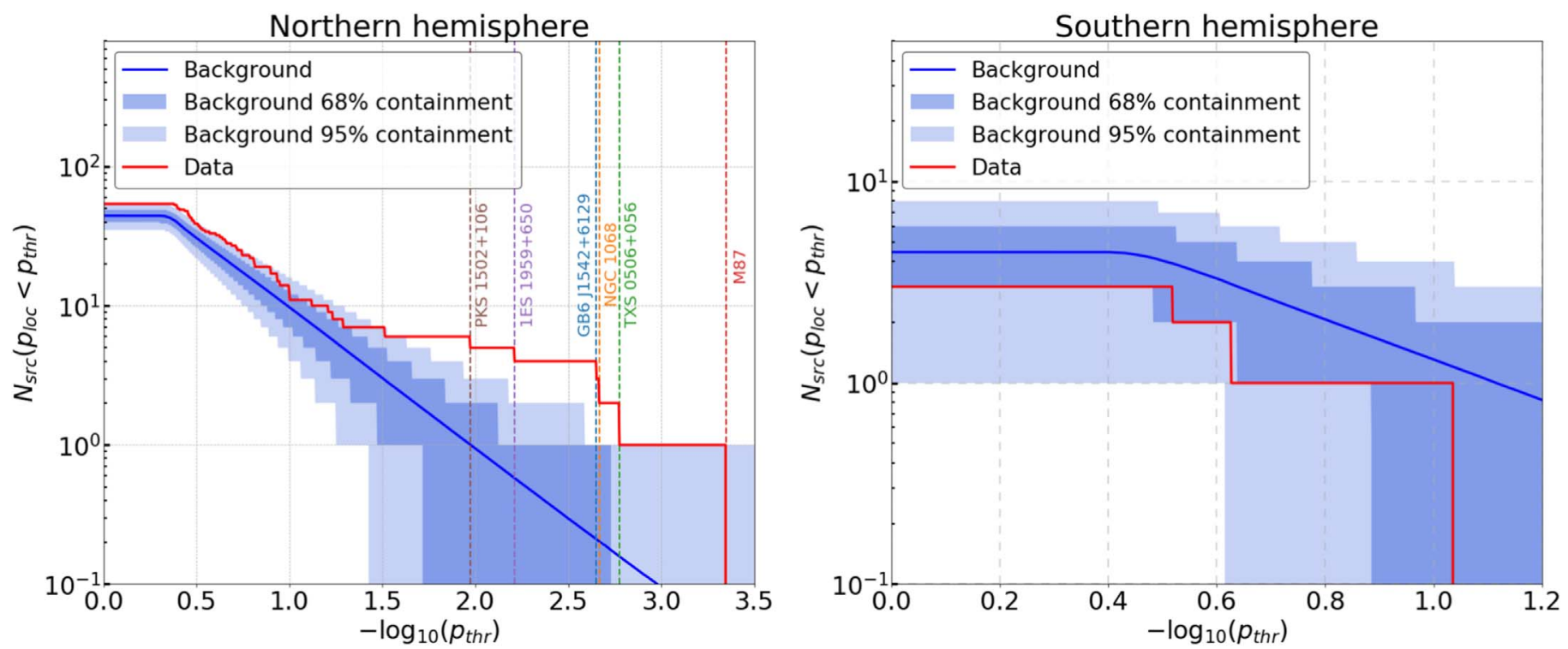

Figure 3. Cumulative distributions of the pre-trial $p$ values of the sources of the catalog in the Northern (left) and Southern (right) hemispheres. The cumulative $p$ values of the unblinded data are shown in red and compared to the background expectations in blue.

\section{Conclusions}

The time-dependent point-source search presented in this Letter identified M87 as the most significant source in the Northern Hemisphere, with $\hat{n}_{s}=3$ signal-like neutrino events in a time window of $\hat{\sigma}_{T}=2.0$ minutes and with a soft spectrum $(\hat{\gamma}=3.95)$. The post-trial significance of M87 is found to be $1.7 \sigma$. Because of the quite short time lag between the events, the time-dependent search is more sensitive than the timeintegrated one, which explains the absence of significant signals in previous IceCube time-integrated analyses that had included M87. For the case of O'Sullivan and Finley (2019), a smaller data sample from 2012 April 26 to 2017 May 11 was used. The difference in significance is due to small changes in the event reconstruction and angular uncertainty estimation between the two samples.

This analysis also identifies the two known flares at the location of TXS $0506+056$, one corresponding to the most significant flare at $\sim 57,000 \mathrm{MJD}$ (Aartsen et al. 2018b) and the other related to the high-energy event alert IceCube-170922A detected on 2017 September 22 (Aartsen et al. 2018a). Although these two flares are consistently identified, the significance of the result at the location of TXS $0506+056$ is lower than the one reported in Aartsen et al. (2018b). This is 

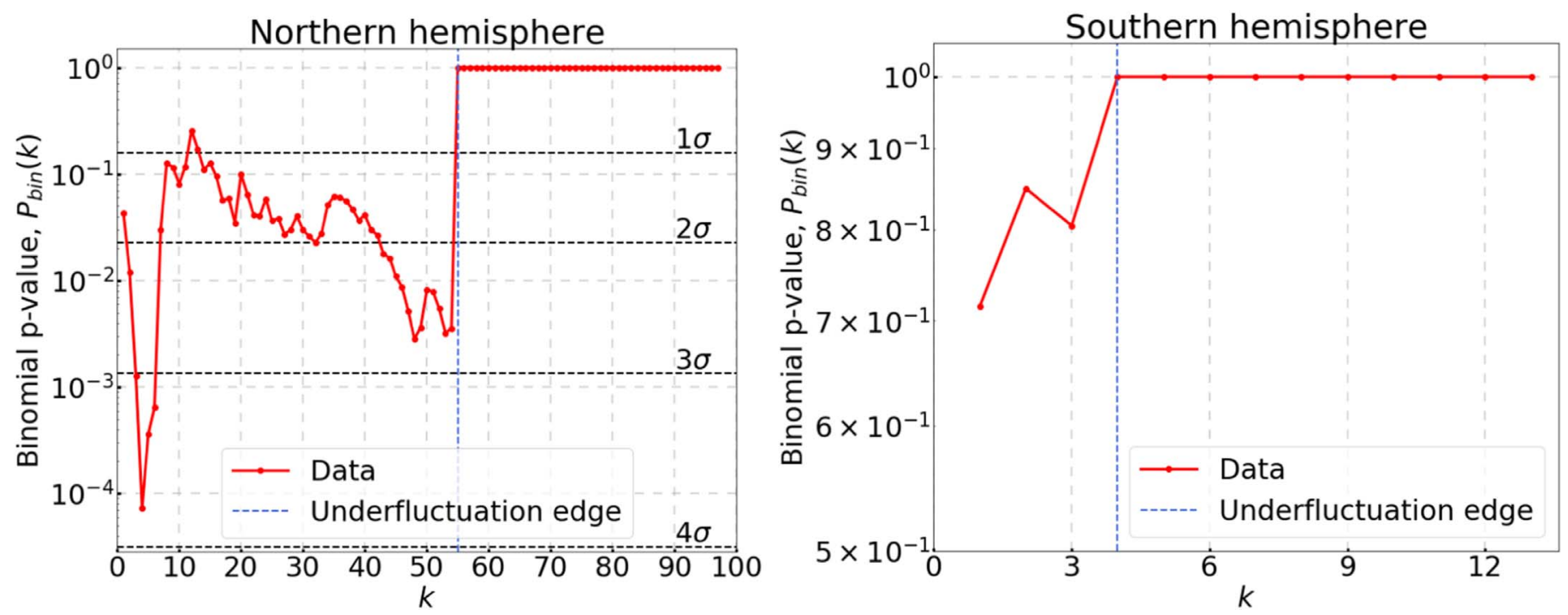

Figure 4. Pre-trial binomial $p$ value $P_{\text {bin }}(k)$ as a function of the source index $k$ in the Northern (left) and Southern (right) hemispheres. The edge with the underfluctuating sources, with binomial $p$ value set to 1 , is shown in blue.

Table 1

Summary of the Results of the Two Analyses

\begin{tabular}{lccc}
\hline \hline \multicolumn{3}{c}{ Summary of the Results } \\
\hline \multirow{2}{*}{ Analysis } & \multirow{2}{*}{ Hemisphere } & \multicolumn{2}{c}{$p$ Value } \\
\cline { 3 - 4 } & & Pre-trial & Post-trial \\
\hline Point-source & North & $4.6 \times 10^{-4}$ & $4.3 \times 10^{-2}(1.7 \sigma)$ \\
& South & $9.2 \times 10^{-2}$ & 0.72 \\
\hline Binomial test & North & $7.3 \times 10^{-5}$ & $1.6 \times 10^{-3}(3.0 \sigma)$ \\
& South & 0.71 & 0.89 \\
\hline
\end{tabular}

Notes. For the point-source search, the results of the best sources in the Northern (M87) and Southern (PKS 2233-148) Hemispheres are reported.

due to the new data selection (Abbasi et al. 2021b) described in Section 2, which introduces a different energy reconstruction from the past one (Abbasi et al. 2021b). Further information about the reduced significance of TXS $0506+056$ resulting from this analysis are provided in Appendix E.

The time-dependent binomial test of the Northern Hemisphere suggests an incompatibility at $3.0 \sigma$ significance of the neutrino events from four sources with respect to the overall Northern background expectation. Of the four most significant sources in the Northern Hemisphere, three are common with the time-integrated analysis (Aartsen et al. 2020c), namely NGC 1068, TXS 0506+056, GB6 J1542+6129, whereas a fourth source (M87) is different and shows a strong timedependent behavior. However, the results of the timedependent and time-integrated binomial test partly overlap, as both share the same space and energy PDFs in the likelihood definition in Equation (2) and both select the same three out of four sources. For this reason, although a time-dependent structure of the data is suggested by the binomial test, a timeindependent scenario cannot be excluded by this analysis (see Appendix $\mathrm{C}$ for a further discussion).

No significant result is found in the Southern Hemisphere. This is consistent with the lower sensitivity due to the substantially larger background of atmospheric muons in the Southern Hemisphere.
The IceCube collaboration gratefully acknowledges the support from the following agencies and institutions: USA-U.S. National Science Foundation-Office of Polar Programs, U.S. National Science Foundation-Physics Division, U.S. National Science Foundation-EPSCoR, Wisconsin Alumni Research Foundation, Center for High Throughput Computing (CHTC) at the University of Wisconsin-Madison, Open Science Grid (OSG), Extreme Science and Engineering Discovery Environment (XSEDE), Frontera computing project at the Texas Advanced Computing Center, U.S. Department of Energy-National Energy Research Scientific Computing Center, Particle astrophysics research computing center at the University of Maryland, Institute for Cyber-Enabled Research at Michigan State University, and Astroparticle physics computational facility at Marquette University; Belgium-Funds for Scientific Research (FRS-FNRS and FWO), FWO Odysseus and Big Science programmes, and Belgian Federal Science Policy Office (Belspo); GermanyBundesministerium für Bildung und Forschung (BMBF), Deutsche Forschungsgemeinschaft (DFG), Helmholtz Alliance for Astroparticle Physics (HAP), Initiative and Networking Fund of the Helmholtz Association, Deutsches Elektronen Synchrotron (DESY), and High Performance Computing cluster of the RWTH Aachen; Sweden-Swedish Research Council, Swedish Polar Research Secretariat, Swedish National Infrastructure for Computing (SNIC), and Knut and Alice Wallenberg Foundation; Australia-Australian Research Council; Canada-Natural Sciences and Engineering Research Council of Canada, Calcul Québec, Compute Ontario, Canada Foundation for Innovation, WestGrid, and Compute Canada; Denmark-Villum Fonden and Carlsberg Foundation; New Zealand-Marsden Fund; Japan-Japan Society for Promotion of Science (JSPS) and Institute for Global Prominent Research (IGPR) of Chiba University; Korea-National Research Foundation of Korea (NRF); Switzerland-Swiss National Science Foundation (SNSF); United Kingdom-Department of Physics, University of Oxford.

\section{Appendix A}

\section{Sensitivity, Discovery Potential, and Upper Limits}

The sensitivity and discovery potential (DP) are evaluated by injecting a fake signal in the data set and looking at the signal- 

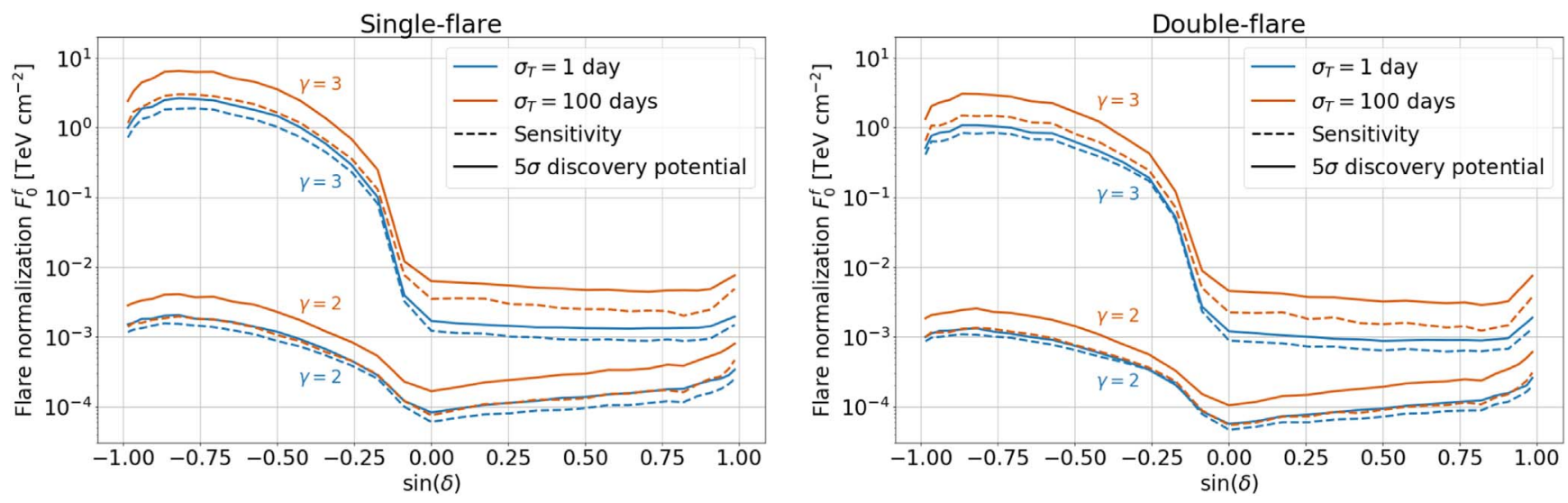

Figure 5. Sensitivity (dashed lines) and $5 \sigma$ DP (solid lines) of the multi-flare analysis vs. decl., expressed in terms of the flux normalization factor per flare $\mathrm{F}_{0}^{f}$ defined in Equation (A1), under the hypothesis of a single (left plot) and a double (right plot) signal flare. The assumed energy dependence of the flares has a spectral index of $\gamma^{f}=2$ and $\gamma^{f}=3$ (see labels), and a flare duration of $\sigma_{T}^{f}=1$ day (blue lines) and $\sigma_{T}^{f}=100$ days (orange lines). The double-flare assumes two identical and wellseparated flares.

like TS distributions. The sensitivity is defined as the signal flux required such that the resulting TS is greater than the background median in $90 \%$ of the trials; the $5 \sigma$ DP is defined as the signal flux required such that the resulting TS is greater than the $5 \sigma$ threshold of the background TS distribution in $50 \%$ of the trials. The sensitivity and $5 \sigma$ discovery potential (DP) of the multi-flare analysis as a function of the decl. are shown in Figure 5 for a single (left) and a double (right) signal flare hypothesis. In the latter case, the intensity and spectral shape of the two flares are the same.

The sensitivity and $5 \sigma$ DP are expressed in terms of a timeintegrated flux:

$$
F=\int_{T_{\text {live }}} E^{2} \Phi(E, t) d t=\sum_{f=\text { flares }} F_{0}^{f}\left(\frac{E}{\mathrm{TeV}}\right)^{2-\gamma^{f}},
$$

where $\mathrm{F}_{0}^{f}$ is the time-integrated flux normalization factor of the $f$ th flare, independent of the flare duration $\sigma_{T}^{f}$ and carrying the units of an energy divided by an area, and $\Phi(E, t)$ is the overall flux, defined as the sum of the flux of all the flares from a single direction:

$$
\Phi(E, t)=\sum_{f=\text { flares }} \frac{F_{0}^{f}}{\sqrt{2 \pi} \sigma_{T}^{f}}\left(\frac{E}{\mathrm{TeV}}\right)^{-\gamma^{f}} G^{f}\left(t \mid t_{0}^{f}, \sigma_{T}^{f}\right)
$$

In Equation (A2), $G^{f}\left(t \mid t_{0}^{f}, \sigma_{T}^{f}\right)=\exp \left[-\frac{1}{2}\left(\frac{t-t_{0}^{f}}{\sigma_{T}^{f}}\right)^{2}\right]$ is the Gaussian time profile of the fth flare.
Sensitivities and DPs are shown in Figure 5 for two different hypotheses of the spectral index of the flares $\left(\gamma^{f}=2\right.$ and $\left.\gamma^{f}=3\right)$ and two different flare durations $\left(\sigma_{T}^{f}=1\right.$ day and $\sigma_{T}^{f}=100$ days). In the double-flare case, two identical and well-separated flares are assumed, with the same spectral index $\gamma^{f}$, flare duration $\sigma_{T}^{f}$ and time-integrated flux normalization per flare $\mathrm{F}_{0}^{f}$

The $90 \%$ confidence level (CL) upper limits on the flux of each source of the catalog are defined as the flux required to produce a TS distribution that exceeds the unblinded TS of the respective source in $90 \%$ of the trials. These upper limits are expressed in terms of a time-integrated flux by means of the factor $F_{90 \%}$, defined as:

$$
F=F_{90 \%} \sum_{f=\text { flares }}\left(\frac{E}{\mathrm{TeV}}\right)^{2-\gamma^{f}} .
$$

In the case of TXS 0506+056, the only observed multi-flare source of the catalog, the upper limits are evaluated assuming the same flare intensity for the two flares. As a matter of fact, only one global factor $F_{90 \%}$ appears in Equation (A3).

The upper limits of the sources of the catalog that are not under-fluctuating, together with the coordinates, maximumlikelihood parameters and pre-trial $p$ values, are reported in Table 2. To calculate these upper limits, a spectral index $\gamma^{f}=2$ in Equation (A3) is assumed for all the flares, whereas the flare time $t_{0}^{f}$ and duration $\sigma_{T}^{f}$ are taken as the ML parameters. Only one flare is injected for each source, except for TXS 0506+056 for which two flares are injected, according to the ML results. 
Table 2

Coordinates, ML Flare Parameters, Logarithm of the Local Pre-trial $p$ Values of the Sources of the Catalog, and the 90\% CL Upper Limits on the Time-integrated Flux Defined in Equation (A3) for an $E^{-2}$ Spectrum

\begin{tabular}{|c|c|c|c|c|c|c|c|c|}
\hline \multicolumn{9}{|c|}{ catalog results } \\
\hline Source & R.A. & $\delta$ & $\hat{n}_{s}$ & $\hat{\gamma}$ & $\hat{t}_{0}$ & $\hat{\sigma}_{T}$ & $-\log _{10}\left(p_{\text {loc }}\right)$ & $F_{90 \%} \times 10^{4}$ \\
\hline & $(\operatorname{deg})$ & $(\operatorname{deg})$ & & & (MJD) & (days ) & & $\left(\mathrm{TeV} \mathrm{cm}{ }^{-2}\right)$ \\
\hline Source & $\begin{array}{l}\text { R.A. } \\
\text { (deg ) }\end{array}$ & $\begin{array}{c}\delta \\
(\operatorname{deg})\end{array}$ & $\hat{n}_{s}$ & $\hat{\gamma}$ & $\begin{array}{c}\hat{t}_{0} \\
(\mathrm{MJD})\end{array}$ & $\begin{array}{c}\hat{\sigma}_{T} \\
\text { (days ) }\end{array}$ & $-\log _{10}\left(p_{\text {loc }}\right)$ & $\begin{array}{c}F_{90 \%} \times 10^{4} \\
\left(\mathrm{TeV} \mathrm{cm}{ }^{-2}\right)\end{array}$ \\
\hline S5 $0716+71$ & 110.49 & 71.34 & $\cdots$ & $\cdots$ & $\cdots$ & $\cdots$ & $\cdots$ & $\cdots$ \\
\hline S4 $1749+70$ & 267.15 & 70.10 & $\ldots$ & $\ldots$ & $\ldots$ & $\ldots$ & $\ldots$ & $\ldots$ \\
\hline M82 & 148.95 & 69.67 & 27.8 & 4.0 & 57395.8 & 200.0 & 1.51 & 5.7 \\
\hline 1ES $1959+650$ & 300.01 & 65.15 & 3.9 & 3.3 & 55028.4 & $1.8 \times 10^{-1}$ & 2.21 & 3.8 \\
\hline GB6 J1542+6129 & 235.75 & 61.50 & $\mathbf{2 3 .} 7_{-7.9}^{+9.7}$ & $\mathbf{2 . 7 _ { - 0 . 3 } ^ { + 0 . 5 }}$ & $\mathbf{5 7 7 4 0}_{-60}^{+80}$ & $\mathbf{1 4 7}_{-25}^{+110}$ & 2.67 & 5.3 \\
\hline PG $1246+586$ & 192.08 & 58.34 & $\cdots$ & $\cdots$ & $\cdots$ & $\cdots$ & $\cdots$ & $\cdots$ \\
\hline TXS $1902+556$ & 285.80 & 55.68 & 3.2 & 4.0 & 54862.5 & 6.0 & 0.46 & 3.6 \\
\hline $4 C+55.17$ & 149.42 & 55.38 & 11.2 & 3.6 & 58303.7 & 59.7 & 1.00 & 2.5 \\
\hline S4 $1250+53$ & 193.31 & 53.02 & 6.1 & 2.2 & 55062.9 & 35.9 & 0.74 & 3.7 \\
\hline 1ES $0806+524$ & 122.46 & 52.31 & 6.5 & 3.1 & 55248.3 & 43.3 & 0.39 & 2.8 \\
\hline $1 \mathrm{H} 1013+498$ & 153.77 & 49.43 & 3.1 & 2.2 & 58053.6 & $2.7 \times 10^{-1}$ & 0.41 & 1.2 \\
\hline B3 $1343+451$ & 206.40 & 44.88 & 4.0 & 2.7 & 57856.5 & $2.8 \times 10^{-1}$ & 0.49 & 1.2 \\
\hline MG4 J200112+4352 & 300.30 & 43.89 & 11.6 & 2.0 & 56776.2 & 105.9 & 1.00 & 2.6 \\
\hline $3 \mathrm{C} 66 \mathrm{~A}$ & 35.67 & 43.04 & $\ldots$ & $\ldots$ & $\ldots$ & $\ldots$ & $\ldots$ & $\ldots$ \\
\hline S4 $0814+42$ & 124.56 & 42.38 & 3.4 & 2.6 & 56301.3 & 3.1 & 0.47 & 1.3 \\
\hline BL Lac & 330.69 & 42.28 & 3.8 & 4.0 & 54637.6 & 5.6 & 0.48 & 2.5 \\
\hline $2 \mathrm{HWC} \mathrm{J} 2031+415$ & 307.93 & 41.51 & 18.8 & 3.4 & 58056.8 & 114.0 & 0.93 & 2.4 \\
\hline NGC 1275 & 49.96 & 41.51 & $\cdots$ & $\cdots$ & $\cdots$ & $\cdots$ & $\cdots$ & $\cdots$ \\
\hline B3 $0609+413$ & 93.22 & 41.37 & 8.7 & 1.7 & 56736.2 & 163.7 & 0.90 & 2.5 \\
\hline M31 & 10.82 & 41.24 & 8.6 & 2.3 & 57900.7 & 16.4 & 1.29 & 2.1 \\
\hline TXS $2241+406$ & 341.06 & 40.96 & 3.8 & 2.9 & 55334.5 & $2.5 \times 10^{-1}$ & 0.55 & 1.7 \\
\hline Gamma Cygni & 305.56 & 40.26 & 5.8 & 1.5 & 57336.8 & 13.0 & 0.95 & 1.8 \\
\hline Mkn 501 & 253.47 & 39.76 & $\ldots$ & $\ldots$ & $\ldots$ & $\ldots$ & $\ldots$ & $\ldots$ \\
\hline B3 $0133+388$ & 24.14 & 39.10 & $\cdots$ & $\ldots$ & $\ldots$ & $\ldots$ & $\cdots$ & $\ldots$ \\
\hline Mkn 421 & 166.12 & 38.21 & 2.9 & 2.1 & 54875.0 & $7.6 \times 10^{-1}$ & 1.23 & 2.8 \\
\hline $4 C+38.41$ & 248.82 & 38.14 & 6.2 & 2.1 & 56751.6 & 9.0 & 0.60 & 1.5 \\
\hline MG2 J201534+3710 & 303.92 & 37.19 & 3.9 & 1.3 & 57326.7 & 129.4 & 0.45 & 1.8 \\
\hline MGRO J2019+37 & 304.85 & 36.80 & 4.2 & 1.4 & 57330.9 & 135.0 & 0.40 & 1.7 \\
\hline B2 $0218+357$ & 35.28 & 35.94 & $\cdots$ & $\cdots$ & $\cdots$ & $\cdots$ & $\cdots$ & $\cdots$ \\
\hline B2 $2114+33$ & 319.06 & 33.66 & $\cdots$ & $\ldots$ & $\ldots$ & $\ldots$ & $\cdots$ & $\ldots$ \\
\hline B2 $1520+31$ & 230.55 & 31.74 & 5.0 & 2.4 & 55999.0 & 2.7 & 0.66 & 1.2 \\
\hline NGC 598 & 23.52 & 30.62 & 4.9 & 1.8 & 56520.7 & 33.0 & 0.75 & 1.7 \\
\hline PG $1218+304$ & 185.34 & 30.17 & 2.0 & 2.4 & 54647.8 & $2.1 \times 10^{-2}$ & 1.12 & 2.1 \\
\hline B2 $1215+30$ & 184.48 & 30.12 & 2.0 & 2.4 & 54647.8 & $2.1 \times 10^{-2}$ & 1.21 & 2.2 \\
\hline Ton 599 & 179.88 & 29.24 & 2.0 & 1.7 & 55024.2 & $3.0 \times 10^{-3}$ & 0.45 & 1.2 \\
\hline MG2 J043337+2905 & 68.41 & 29.10 & $\cdots$ & $\ldots$ & $\cdots$ & $\ldots$ & $\cdots$ & $\ldots$ \\
\hline $4 C+28.07$ & 39.48 & 28.80 & $\cdots$ & $\cdots$ & $\cdots$ & $\cdots$ & $\cdots$ & $\cdots$ \\
\hline W Comae & 185.38 & 28.24 & 3.1 & 3.4 & 55682.4 & 1.5 & 0.49 & 1.2 \\
\hline TXS $0141+268$ & 26.15 & 27.09 & $\cdots$ & $\cdots$ & $\ldots$ & $\ldots$ & $\ldots$ & $\ldots$ \\
\hline ON 246 & 187.56 & 25.30 & $\cdots$ & $\cdots$ & $\cdots$ & $\cdots$ & $\cdots$ & $\cdots$ \\
\hline 1ES $0647+250$ & 102.70 & 25.06 & $\cdots$ & $\cdots$ & $\cdots$ & $\cdots$ & $\cdots$ & $\cdots$ \\
\hline PKS $1441+25$ & 220.99 & 25.03 & 4.1 & 1.7 & 56994.7 & 105.6 & 0.69 & 1.8 \\
\hline PKS $1424+240$ & 216.76 & 23.80 & 17.9 & 2.8 & 57182.6 & 200.0 & 1.00 & 2.2 \\
\hline S2 $0109+22$ & 18.03 & 22.75 & 4.6 & 4.0 & 55153.2 & $9.2 \times 10^{-1}$ & 0.93 & 1.6 \\
\hline Crab Nebula & 83.63 & 22.01 & $\ldots$ & $\ldots$ & $\ldots$ & $\ldots$ & $\cdots$ & $\ldots$ \\
\hline $4 C+21.35$ & 186.23 & 21.38 & 2.0 & 2.3 & 55690.3 & $1.2 \times 10^{-3}$ & 0.64 & 0.9 \\
\hline TXS $0518+211$ & 80.44 & 21.21 & $\ldots$ & $\ldots$ & $\ldots$ & $\ldots$ & $\ldots$ & $\ldots$ \\
\hline RGB J2243+203 & 340.99 & 20.36 & 11.2 & 3.6 & 57300.1 & 33.0 & 0.81 & 1.5 \\
\hline OJ 287 & 133.71 & 20.12 & 3.6 & 2.6 & 56416.8 & $8.4 \times 10^{-1}$ & 0.72 & 1.0 \\
\hline PKS $1717+177$ & 259.81 & 17.75 & 2.0 & 3.3 & 54587.2 & $2.0 \times 10^{-1}$ & 0.45 & 1.3 \\
\hline OX 169 & 325.89 & 17.73 & $\ldots$ & $\ldots$ & $\ldots$ & $\ldots$ & $\ldots$ & $\ldots$ \\
\hline PKS $0735+17$ & 114.54 & 17.71 & $\ldots$ & $\ldots$ & $\ldots$ & $\ldots$ & $\ldots$ & $\ldots$ \\
\hline PKS $0235+164$ & 39.67 & 16.62 & $\cdots$ & $\cdots$ & $\cdots$ & $\cdots$ & $\cdots$ & $\cdots$ \\
\hline 3C 454.3 & 343.50 & 16.15 & 5.1 & 2.0 & 56119.1 & 200.0 & 0.46 & 1.3 \\
\hline $4 C+14.23$ & 111.33 & 14.42 & 3.1 & 2.0 & 58076.6 & 1.2 & 0.81 & 1.0 \\
\hline PSR B0656+14 & 104.95 & 14.24 & $\cdots$ & $\cdots$ & $\cdots$ & $\ldots$ & $\cdots$ & $\cdots$ \\
\hline M87 & 187.71 & 12.39 & $\mathbf{3 . 0}_{-1.4}^{+2.0}$ & $\mathbf{4 . 0 _ { - 0 . 9 } ^ { + 0 . 9 }}$ & $\mathbf{5 7 7 3 0 . 0 3 1}_{-0.001}^{+0.001}$ & $\mathbf{1 . 4}_{-0.4}^{+1.3} \times \mathbf{1 0}^{-3}$ & 3.35 & 0.9 \\
\hline $1 \mathrm{H} 1720+117$ & 261.27 & 11.88 & $\ldots$ & $\ldots$ & $\ldots$ & $\ldots$ & $\cdots$ & $\ldots$ \\
\hline CTA 102 & 338.15 & 11.73 & $\ldots$ & $\ldots$ & $\ldots$ & $\ldots$ & $\ldots$ & $\ldots$ \\
\hline
\end{tabular}


Table 2

(Continued)

\begin{tabular}{|c|c|c|c|c|c|c|c|c|}
\hline \multicolumn{9}{|c|}{ catalog results } \\
\hline Source & R.A. & $\delta$ & $\hat{n}_{s}$ & $\hat{\gamma}$ & $\hat{t}_{0}$ & $\hat{\sigma}_{T}$ & $-\log _{10}\left(p_{\text {loc }}\right)$ & $F_{90 \%} \times 10^{4}$ \\
\hline PG $1553+113$ & 238.93 & 11.19 & $\ldots$ & $\ldots$ & $\ldots$ & $\ldots$ & $\ldots$ & $\ldots$ \\
\hline PKS $2032+107$ & 308.85 & 10.94 & $\ldots$ & $\ldots$ & $\ldots$ & $\ldots$ & $\ldots$ & $\ldots$ \\
\hline MG1 J021114+1051 & 32.81 & 10.86 & 2.8 & 2.1 & 56179.2 & $8.9 \times 10^{-1}$ & 0.52 & 0.9 \\
\hline 1RXS J194246.3+1 & 295.70 & 10.56 & 4.2 & 3.4 & 54904.8 & 24.3 & 0.51 & 1.4 \\
\hline PKS $1502+106$ & 226.10 & 10.50 & 9.8 & 2.5 & 55509.5 & 21.6 & 1.97 & 1.8 \\
\hline OT 081 & 267.87 & 9.65 & 9.7 & 2.9 & 57751.6 & 45.7 & 0.79 & 1.3 \\
\hline RX J1931.1+0937 & 292.78 & 9.63 & $\ldots$ & $\ldots$ & $\ldots$ & $\ldots$ & $\ldots$ & $\ldots$ \\
\hline $\mathrm{OG}+050$ & 83.18 & 7.55 & $\ldots$ & $\ldots$ & $\ldots$ & $\ldots$ & $\ldots$ & $\ldots$ \\
\hline MGRO J1908+06 & 287.17 & 6.18 & 2.9 & 2.1 & 57045.2 & 4.6 & 0.63 & 0.9 \\
\hline PKS 0019+058 & 5.64 & 6.14 & $\ldots$ & $\ldots$ & $\ldots$ & $\ldots$ & $\ldots$ & $\ldots$ \\
\hline \multirow[t]{2}{*}{ TXS 0506+056 } & 77.35 & 5.70 & $\mathbf{1 0 . 0 _ { - 4 . 2 } ^ { + 5 . 2 }}$ & $\mathbf{2 . 2 _ { - 0 . 3 } ^ { + 0 . 3 }}$ & $\mathbf{5 7 0 0 0}_{-30}^{+30}$ & $\mathbf{6 2}_{-27}^{+27}$ & 2.77 & 1.7 \\
\hline & & & $\mathbf{7 . 6}_{-5.8}^{+6.1}$ & $\mathbf{2 . 6}_{-0.6}^{+0.5}$ & $\mathbf{5 8 0 2 0}_{-40}^{+40}$ & $\mathbf{4 2}_{-28}^{+42}$ & & \\
\hline PKS $0502+049$ & 76.34 & 5.00 & 2.7 & 2.0 & 57072.1 & 1.2 & 0.81 & 0.9 \\
\hline MG1 J123931+0443 & 189.89 & 4.73 & $\ldots$ & $\ldots$ & $\ldots$ & $\ldots$ & $\ldots$ & $\ldots$ \\
\hline PKS 0829+046 & 127.97 & 4.49 & $\ldots$ & $\ldots$ & $\ldots$ & $\ldots$ & $\ldots$ & $\ldots$ \\
\hline PKS $1502+036$ & 226.26 & 3.44 & 2.0 & 2.9 & 54606.9 & $3.4 \times 10^{-1}$ & 0.53 & 1.2 \\
\hline HESS J1857+026 & 284.30 & 2.67 & 3.6 & 2.3 & 54984.4 & $2.0 \times 10^{-1}$ & 0.71 & 0.9 \\
\hline $3 \mathrm{C} 273$ & 187.27 & 2.04 & $\ldots$ & $\ldots$ & $\ldots$ & $\ldots$ & $\ldots$ & $\ldots$ \\
\hline OJ 014 & 122.87 & 1.78 & $\ldots$ & $\ldots$ & $\ldots$ & $\ldots$ & $\ldots$ & $\ldots$ \\
\hline PKS $0215+015$ & 34.46 & 1.74 & $\ldots$ & $\ldots$ & $\ldots$ & $\ldots$ & $\ldots$ & $\ldots$ \\
\hline PKS $0736+01$ & 114.82 & 1.62 & $\ldots$ & $\ldots$ & $\ldots$ & $\ldots$ & $\ldots$ & $\ldots$ \\
\hline $4 \mathrm{C}+01.02$ & 17.16 & 1.59 & $\ldots$ & $\ldots$ & $\ldots$ & $\ldots$ & $\ldots$ & $\ldots$ \\
\hline $4 \mathrm{C}+01.28$ & 164.61 & 1.56 & $\ldots$ & $\ldots$ & $\ldots$ & $\ldots$ & $\ldots$ & $\ldots$ \\
\hline GRS 1285.0 & 283.15 & 0.69 & 6.5 & 2.8 & 54808.6 & 87.3 & 0.39 & 1.9 \\
\hline PKS $0422+00$ & 66.19 & 0.60 & $\ldots$ & $\ldots$ & $\ldots$ & $\ldots$ & $\ldots$ & $\ldots$ \\
\hline PKS B1130+008 & 173.20 & 0.58 & $\ldots$ & $\ldots$ & $\ldots$ & $\ldots$ & $\ldots$ & $\ldots$ \\
\hline PMN J0948+0022 & 147.24 & 0.37 & 2.0 & 2.4 & 55610.7 & $4.3 \times 10^{-4}$ & 0.90 & 0.6 \\
\hline HESS J1852-000 & 283.00 & 0.00 & 5.4 & 2.8 & 54751.9 & 100.3 & 0.38 & 1.9 \\
\hline NGC 1068 & 40.67 & -0.01 & $\mathbf{2 3 . 0}_{-7.9}^{+8.7}$ & $\mathbf{2 . 8}_{-0.3}^{+0.3}$ & $\mathbf{5 6 2 9 0}_{-80}^{+90}$ & $\mathbf{1 9 8}_{-64}^{+64}$ & 2.65 & 1.9 \\
\hline HESS J1849-000 & 282.26 & -0.02 & $\ldots$ & $\ldots$ & $\ldots$ & $\ldots$ & $\ldots$ & $\ldots$ \\
\hline PKS 0440-00 & 70.66 & -0.29 & 6.2 & 2.6 & 57896.8 & 66.8 & 0.51 & 0.9 \\
\hline PKS 1216-010 & 184.64 & -1.33 & $\ldots$ & $\ldots$ & $\ldots$ & $\ldots$ & $\ldots$ & $\ldots$ \\
\hline PKS 0420-01 & 65.83 & -1.33 & $\ldots$ & $\ldots$ & $\ldots$ & $\ldots$ & $\ldots$ & $\ldots$ \\
\hline NVSS J190836-012 & 287.20 & -1.53 & $\ldots$ & $\ldots$ & $\ldots$ & $\ldots$ & $\ldots$ & $\ldots$ \\
\hline PKS 0336-01 & 54.88 & -1.77 & $\ldots$ & $\ldots$ & $\ldots$ & $\ldots$ & $\ldots$ & $\ldots$ \\
\hline S3 $0458-02$ & 75.30 & -1.97 & 4.6 & 2.5 & 56974.6 & $7.0 \times 10^{-1}$ & 0.65 & 0.7 \\
\hline NVSS J141826-023 & 214.61 & -2.56 & 3.7 & 2.9 & 57733.0 & $3.4 \times 10^{-1}$ & 0.44 & 0.6 \\
\hline PKS 2320-035 & 350.88 & -3.29 & 10.8 & 3.2 & 56176.8 & 160.2 & 0.57 & 1.1 \\
\hline HESS J1843-033 & 280.75 & -3.30 & $\cdots$ & $\cdots$ & $\cdots$ & $\cdots$ & $\cdots$ & $\cdots$ \\
\hline PKS 1329-049 & 203.02 & -5.16 & $\ldots$ & $\ldots$ & $\ldots$ & $\ldots$ & $\ldots$ & $\ldots$ \\
\hline HESS J1841-055 & 280.23 & -5.55 & $\ldots$ & $\ldots$ & $\ldots$ & $\ldots$ & $\ldots$ & $\ldots$ \\
\hline $3 C 279$ & 194.04 & -5.79 & $\ldots$ & $\ldots$ & $\ldots$ & $\cdots$ & $\cdots$ & $\cdots$ \\
\hline HESS J1837-069 & 279.43 & -6.93 & $\ldots$ & $\ldots$ & $\ldots$ & $\ldots$ & $\ldots$ & $\ldots$ \\
\hline PKS 0805-07 & 122.07 & -7.86 & $\ldots$ & $\ldots$ & $\ldots$ & $\ldots$ & $\ldots$ & $\ldots$ \\
\hline PKS $1510-089$ & 228.21 & -9.10 & $\ldots$ & $\ldots$ & $\ldots$ & $\ldots$ & $\ldots$ & $\ldots$ \\
\hline PKS 0048-09 & 12.68 & -9.49 & $\ldots$ & $\ldots$ & $\ldots$ & $\ldots$ & $\ldots$ & $\ldots$ \\
\hline PKS 0727-11 & 112.58 & -11.69 & $\ldots$ & $\ldots$ & $\ldots$ & $\ldots$ & $\ldots$ & $\ldots$ \\
\hline PKS 2233-148 & 339.14 & -14.56 & 2.0 & 2.8 & 54877.5 & $2.6 \times 10^{-3}$ & 1.04 & 12.0 \\
\hline NGC 253 & 11.90 & -25.29 & 4.1 & 2.5 & 56511.7 & 22.7 & 0.52 & 8.7 \\
\hline NGC 4945 & 196.36 & -49.47 & 2.0 & 1.9 & 54739.8 & $2.4 \times 10^{-1}$ & 0.63 & 55.3 \\
\hline LMC & 80.00 & -68.75 & $\ldots$ & $\ldots$ & $\ldots$ & $\ldots$ & $\ldots$ & $\ldots$ \\
\hline SMC & 14.50 & -72.75 & $\ldots$ & $\ldots$ & $\ldots$ & $\ldots$ & $\ldots$ & $\ldots$ \\
\hline
\end{tabular}

Note. Under-fluctuating results are shown with hyphens. For the four sources that give rise to the $3.0 \sigma$ excess of the binomial test in the Northern Hemisphere (highlighted in bold), the fit parameters are shown with the confidence interval at 68\% CL. A line is used to separate the Northern from Southern sources. The parameters of the flare from TXS $0506+056$ at $58020 \mathrm{MJD}$ and related to the neutrino alert ( $n_{s}=7.6, \gamma=2.6, \sigma_{T}=42$ days) are different from those reported in Aartsen et al. (2018b) when the data available for analysis extended up to 40 days after the central time of the flare. This analysis includes 7 additional months and reconstructs a longer, more significant flare associated with the same alert.

(This table is available in its entirety in machine-readable form.) 


\section{Appendix B Multi-flare Algorithm}

The multi-flare algorithm aims at determining the number of flares to fit in the data. This is done by evaluating the TS of clusters of events with the highest signal-over-background ratio of the spatial and energy PDFs and selecting as candidate flares those that pass a given TS threshold. On the one hand, a high value of TS threshold is required to suppress background fluctuations (fake flares); however, a low value is desired to avoid the rejection of signal flares of low intensity.

This multi-flare algorithm selects as candidate flares the cluster of events with the highest TS and all additional clusters of events passing a TS threshold of 2. The choice of this threshold ensures a high efficiency in rejecting fake flares with a frequency of multiple flare reconstruction under the null hypothesis of $\lesssim 0.1 \%$ as shown in Figure 6. Such a high rejection efficiency allows us to preserve a sensitivity and a DP comparable to the single-flare algorithm, as shown in Figure 7 at the decl. of TXS $0506+056$. Note additionally that if only one candidate flare is selected by the multi-flare algorithm, the multi-flare algorithm is completely equivalent to the singleflare algorithm.

To quantify the goodness of the multi-flare reconstruction, two quantities are introduced: the multi-flare efficiency, defined as the fraction of trials in which all the signal flares are identified (regardless of whether additional fake flares are also reconstructed), and the multi-flare purity, defined as the fraction of trials in which no fake flares are reconstructed (regardless of whether all the signal flares are identified). The former is an indicator of how frequent the algorithm is able to identify all the signal flares injected in the data; the latter is an indicator of how well the algorithm is able to reject fake flares. Note that a partially reconstructed flare is considered a fake flare in the estimation of efficiency and purity. These two quantities are shown in Figure 8 under the hypothesis of two flares of equal

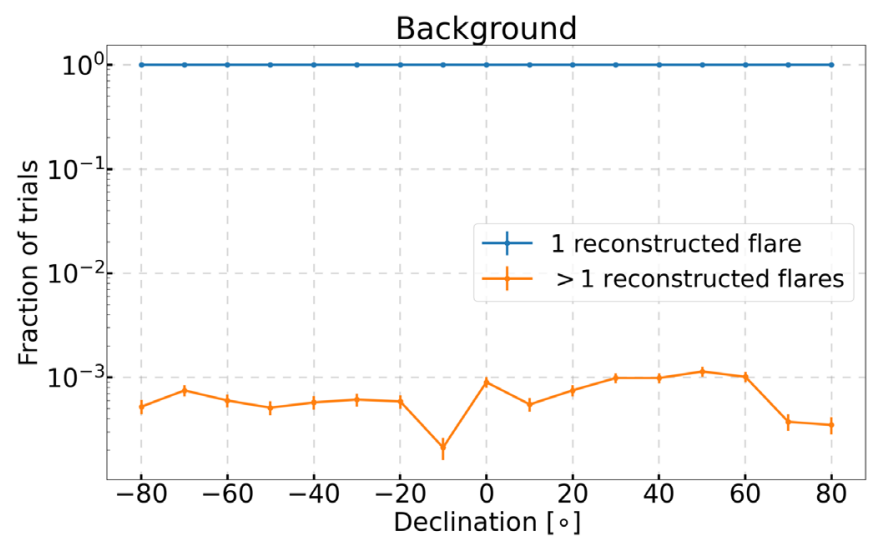

Figure 6. Fraction of trials in which, under the null hypothesis, a single flare (blue line) or more than one flare (orange line) are reconstructed as a function of the decl. if a TS threshold of two is applied to select the candidate flares.
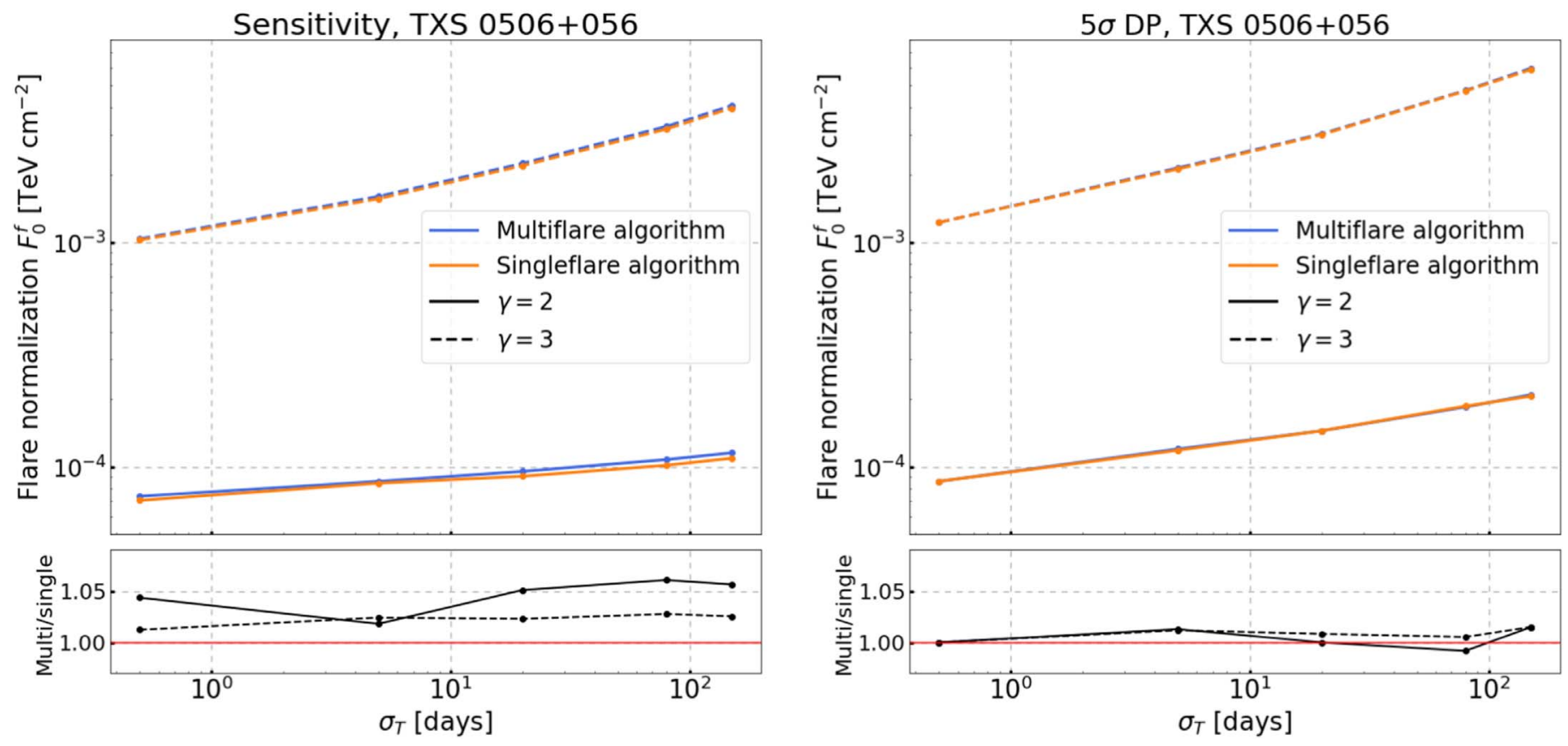

Figure 7. Sensitivity (left) and discovery potential (right) of the single-flare (orange lines) and multi-flare (blue lines) algorithm as a function of the flare duration $\sigma_{T}$. Sensitivity and discovery potential are evaluated at the decl. of TXS $0506+056$ under the hypothesis of a single signal flare with a spectrum $E^{-2}$ (solid lines) and $E^{-3}$ (dashed lines). The bottom plots show the ratio of the multi-flare to single-flare curves above. 

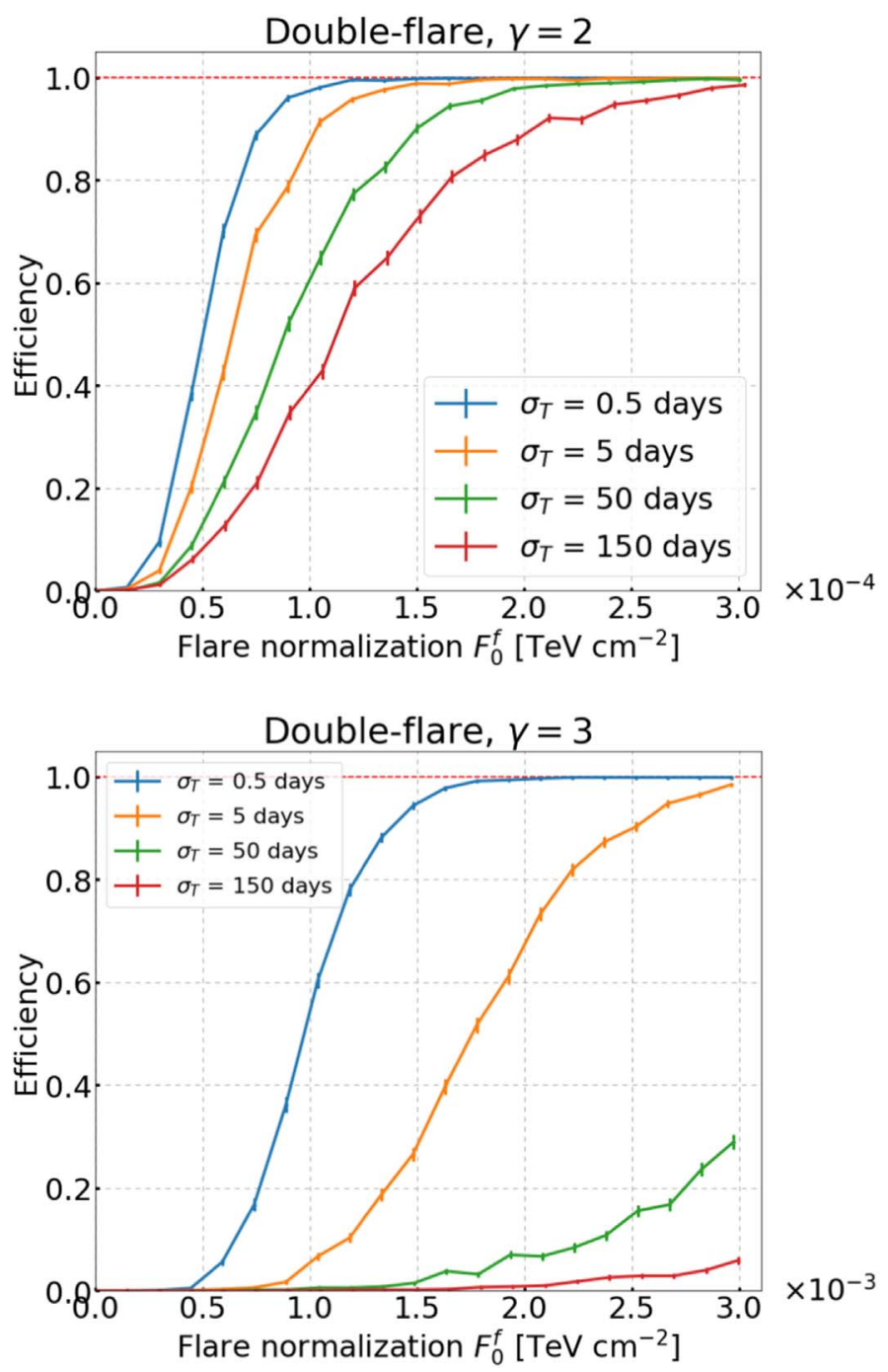
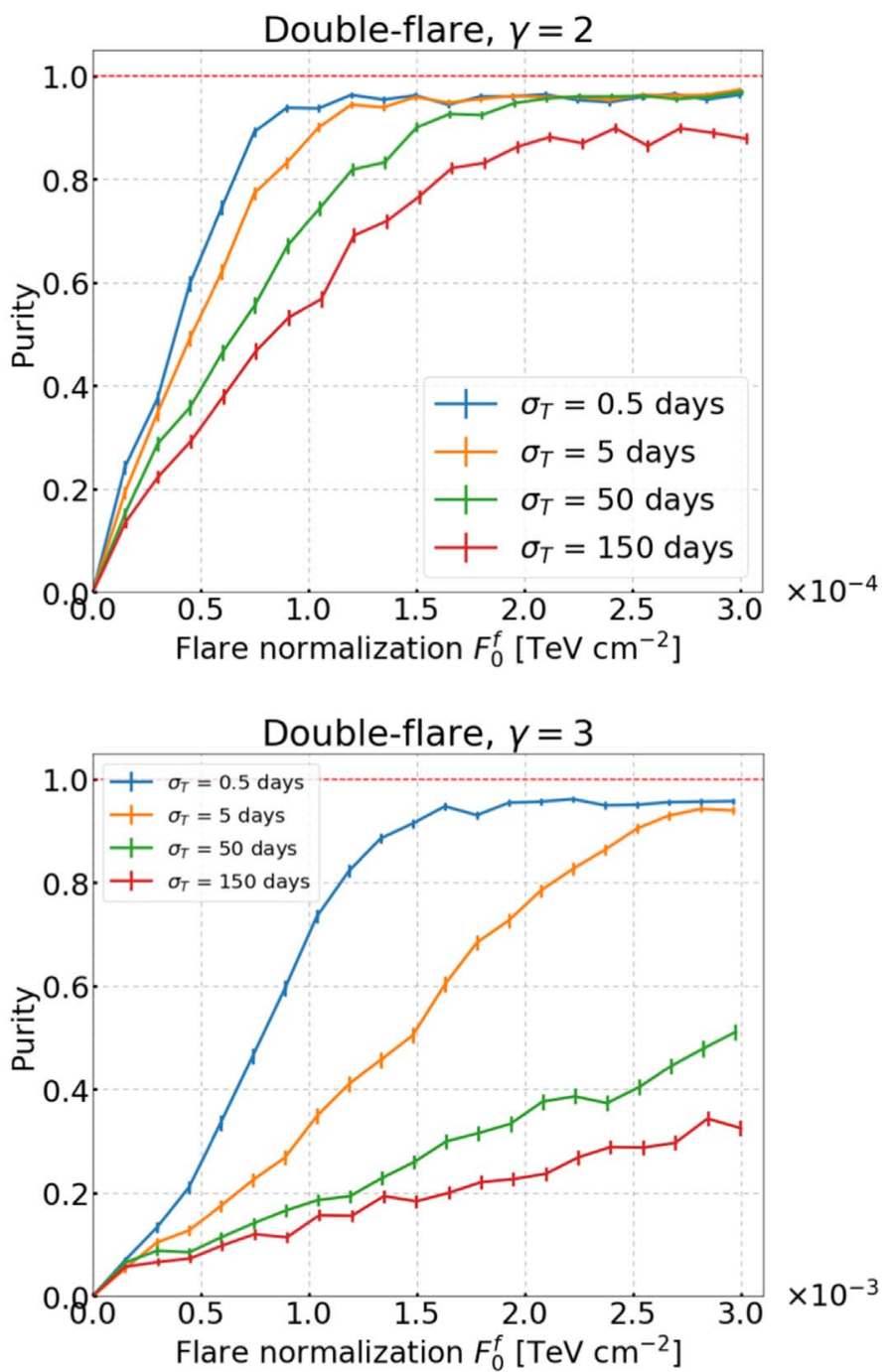

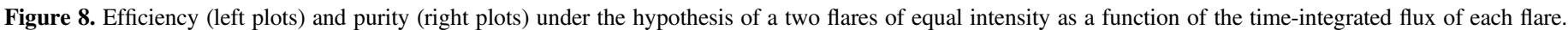

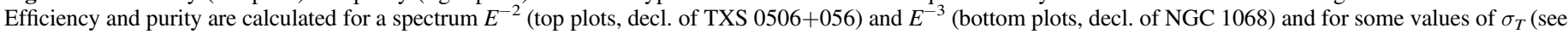

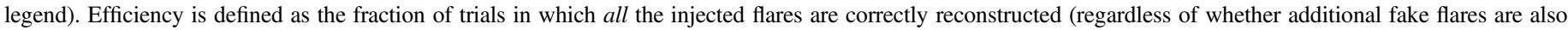

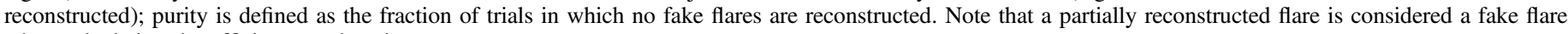
when calculating the efficiency and purity.

intensity as a function of the time-integrated flux of each flare, for spectra $E^{-2}$ and $E^{-3}$ and for some values of $\sigma_{T}$. The efficiency asymptotically reaches the value of 1 : if the signal is strong enough, the algorithm is always able to identify it. However, the flux required to reach such an asymptotic plateau depends on the parameters of the flare (spectral index $\gamma$ and flare duration $\sigma_{T}$ ) and notably, in extreme cases (soft spectra, long flare duration) the convergence is very slow, as a consequence of the high TS threshold. Nevertheless, note that in such extreme cases the flare intensity is mostly below the sensitivity level. The purity also tends toward an asymptotic plateau at $\gtrsim 95 \%$ with a rapidity that depends on the flare parameters.

\section{Appendix C \\ A Posteriori Comparisons with the Time-integrated Analysis}

The results of these time-dependent analyses, despite unveiling new features of the source catalog, partly overlap with the results of the time-integrated search (Aartsen et al. 2020c). In fact, the time-dependent and time-integrated analyses are based on similar likelihood functions, sharing the same space and energy PDFs, but the time-dependent analysis distinguishes itself by adding a time PDF. This time-dependent analysis was planned together with the time-integrated analysis and it was not developed based on the time-integrated unblinded results. Nonetheless, one might wonder how the results of the time-dependent analysis can be interpreted in the light of the prior knowledge of the time-integrated results. To address such a question, two tests are proposed in this Appendix. A first test estimates the time variability of the four most significant sources of the time-integrated analysis. A second test estimates the probability of obtaining the observed pre-trial significance of $3.8 \sigma$ from a time-dependent binomial test (see Section 4) on the source catalog, in the assumption that the neutrino excess observed by the time-integrated analysis (Aartsen et al. 2020c) does not have any time structure. Both tests exploit the same approach, based on producing pseudo-realizations of the data by randomizing the time of the events and, unlike for the standard time-dependent analysis, keeping the associated equatorial coordinates fixed. 
Time-variability test: This test aims at quantifying the time variability of the highly significant events detected from the directions of NGC 1068, TXS 0506+056, PKS 1424+240, and GB6 J1542+6129 and at testing the compatibility of their arrival time with a flat distribution. This test is sensitive only to the time information of the events and is unavoidably less sensitive than the time-dependent search described in Section 3 (referred to as standard time-dependent analysis), which is sensitive to energy, space, and time information. Moreover, the significance of the likelihood method using the three variables at the same time is not equivalent to the product of likelihood methods that use one variable at a time.

The null (or background) hypothesis of the time-variability test assumes that the time-integrated signal-like events (i.e.,the events with the highest time-integrated signal-over-background ratio, that mostly contribute to the significance around each source direction) are not clustered in time. Pseudo-realizations of the data for this null hypothesis (also called background samples, which allow us to count trials) are obtained similarly to the standard time-dependent analysis: events in a decl. band around the location of the tested sources are selected and assigned a new time taken from a real up time of the detector. This procedure destroys any time correlation among events. However, while the standard analysis keeps the local coordinates of an event (azimuth and zenith) fixed and recalculates the R.A. using the new randomized time, the time-variability test freezes the equatorial coordinates of the events at the measured values, and randomizes the azimuth (notably the zenith angle, corresponding to an equatorial coordinate, at the South Pole does not depend on the time). This method guarantees that the same time-integrated signallike events from the direction of a given source are present in the background sample with randomized times. On the other hand, this method flattens out the sub-daily modulation of the event rate in local coordinates due to the increased reconstruction efficiency along the azimuth directions where more strings are aligned. As described in Section 3, in the standard analysis, this sub-daily modulation of the event rate is taken into account by using a correction in local coordinates to the background PDF. The azimuth dependency of the reconstruction efficiency is averaged out for flares longer than $\sigma_{T}=0.2$ days as a consequence of the Earth rotation, while it might induce a change up to 5\% in the TS for flares as short as $\sigma_{T}=10^{-3}$ days. Given that the variability observed for the four most significant time-integrated sources was beyond a flare duration of $\sigma_{T} \gg 1$ day, a lower limit $\sigma_{T}^{\min }=0.2$ days is used for this timevariability test.

Whereas for the standard analysis, signal samples are produced by injecting Monte Carlo events on top of the background events, for the time-variability test $n_{s}$ events among real signal-like events are selected in the data and their times are sampled from a Gaussian distribution. The real signal-like events, potentially usable for signal injection in the time-variability test, are randomly chosen among the $2 \hat{N}_{s}^{t-\text { int }}$ events with the highest time-integrated signal-over-background ratio, where $\hat{N}_{s}^{t-\text { int }}$ is the best-fit number of signal-like events reported by the time-integrated analysis (Aartsen et al. 2020c).

The likelihood in Equation (1) is maximized on the background and signal samples of the time-variability test and the corresponding TS distributions (for illustration at the location of NGC 1068) are shown in Figure 9, for comparison with the same distributions for the standard analysis. For both analyses, the separation of the signal and background TS is better for shorter flares (left plots) than longer ones (right plots). A notable feature concerns the background TS distributions in blue. For the standard analysis, the TS distribution has a characteristic spike in the first bin populated by under-fluctuations set to zero. On the other hand, the TS distribution for the time-variability test is on average shifted toward larger values of TS, showing a more signal-like behavior. This is a consequence of preserving the same timeintegrated space and energy variables of signal-like events in the background sample with the method described above. It is to be noted that the time-integrated analysis in Aartsen et al. (2020c) fits a spectral index of NGC 1068 of 3.16, while the best-fit spectral index for the time-dependent analysis is harder, namely 2.8 (see Table 2). As a consequence of preserving the spatial and energy information of the events, the background and signal samples of the time-variability test (used to make the distributions in the last row in Figure 9) have a varying spectral index centered around 2.8. Notably, about $89 \%$ of the spectral indices of the 100,000 generated background samples are contained between $\gamma^{f}=2$ and of $\gamma^{f}=3$. Hence, these values of the spectral indices are used for the signal injection in the standard analysis when comparing the TS distributions of the standard analysis with the same distributions of the timevariability test in Figure 9. In general, for harder spectral indices and the same flare duration $\sigma_{T}^{f}$, the time-variability test characterizes the difference between background and signal less powerfully than the standard analysis. In fact, in the timevariability test the coordinates of the events are frozen to the true values, hence the differences between the spatial and energy PDFs of signal and background are not exploited, unlike for the standard analysis.

To complete the comparison between the standard timedependent analysis and the time-variability test, the sensitivity and $5 \sigma$ DP at the location of NGC 1068 are shown for the two analyses in Figure 10. The times of signal events are sampled from a Gaussian distribution with fixed mean $t_{0}=58,000 \mathrm{MJD}$ and variable width $\sigma_{T}$. This plot can be understood by observing the TS distributions in Figure 9.

For each of the four aforementioned sources, the likelihood in Equation (1) is maximized on the real data and an observed TS is reported. A pre-trial $p$ value for the time-variability test is then evaluated by counting the fraction of generated background samples with TS larger than the observed TS. The posttrial $p$ value of each source is obtained by applying a Sidak correction (Abdi 2007) to the pre-trial $p$ values with penalty factor 4 (the number of sources). The results of this test are shown in Table 3. None of the four sources shows a significant time variability for the signal-like neutrino events.

It is worth noting the case of M87: this source was an underfluctuation for the time-integrated analysis, with no signal-like events identified in Aartsen et al. (2020c), but it shows up as the most significant source of the catalog when a timedependent analysis is performed. Although a time-variability test is not made in this case, with $\hat{n}_{s}=3$ signal-like neutrino events in a timescale of $\hat{\sigma}_{T}=2.0$ minutes, almost the entire significance of this source is expected to come from the time variability of the detected events.

Posterior time-dependent binomial test: The second test determines the a posteriori probability that the time-dependent binomial test (see Section 3 referred to as "standard" in this Appendix) produces a pre-trial significance as high or higher 
NGC 1068

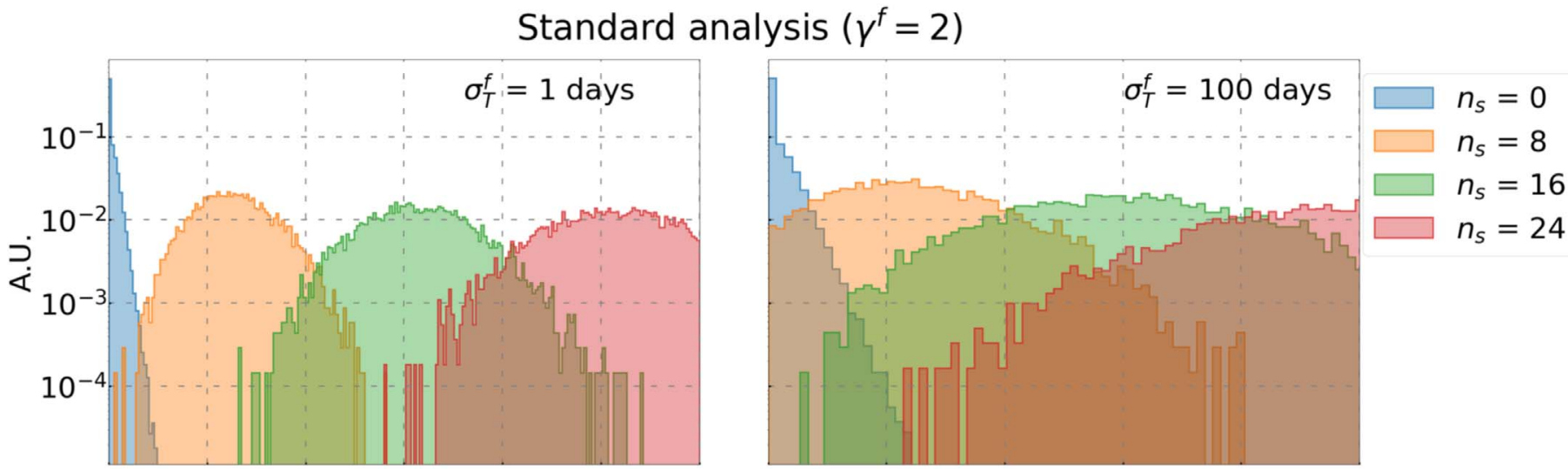

Standard analysis $\left(\gamma^{f}=3\right)$
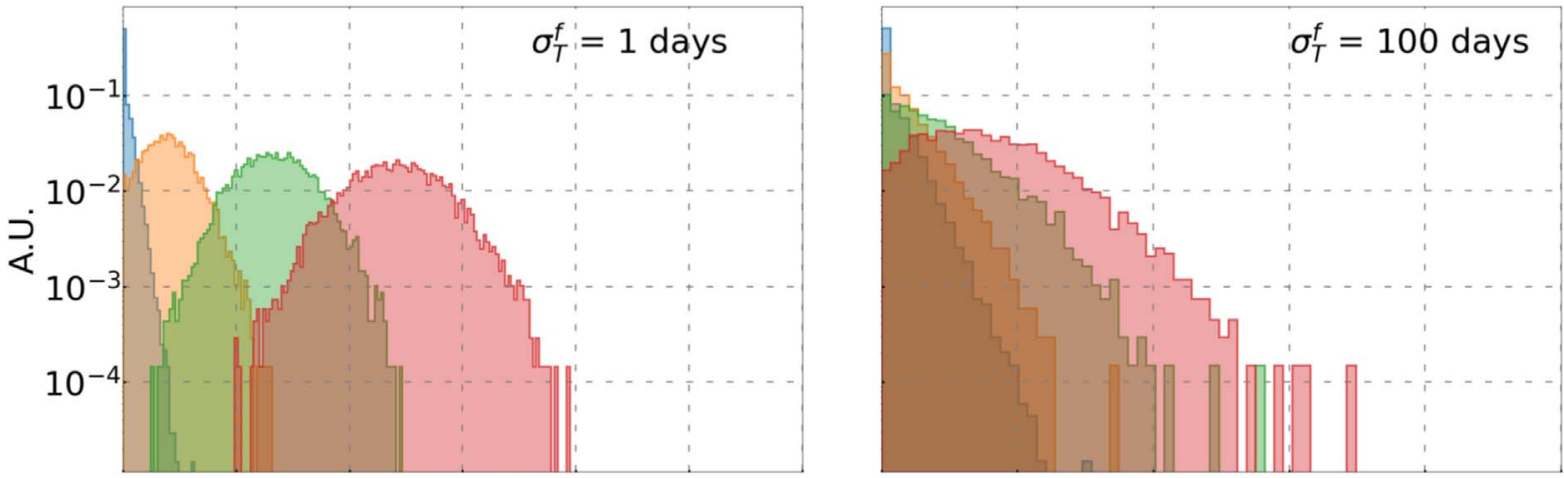

Time-variability test
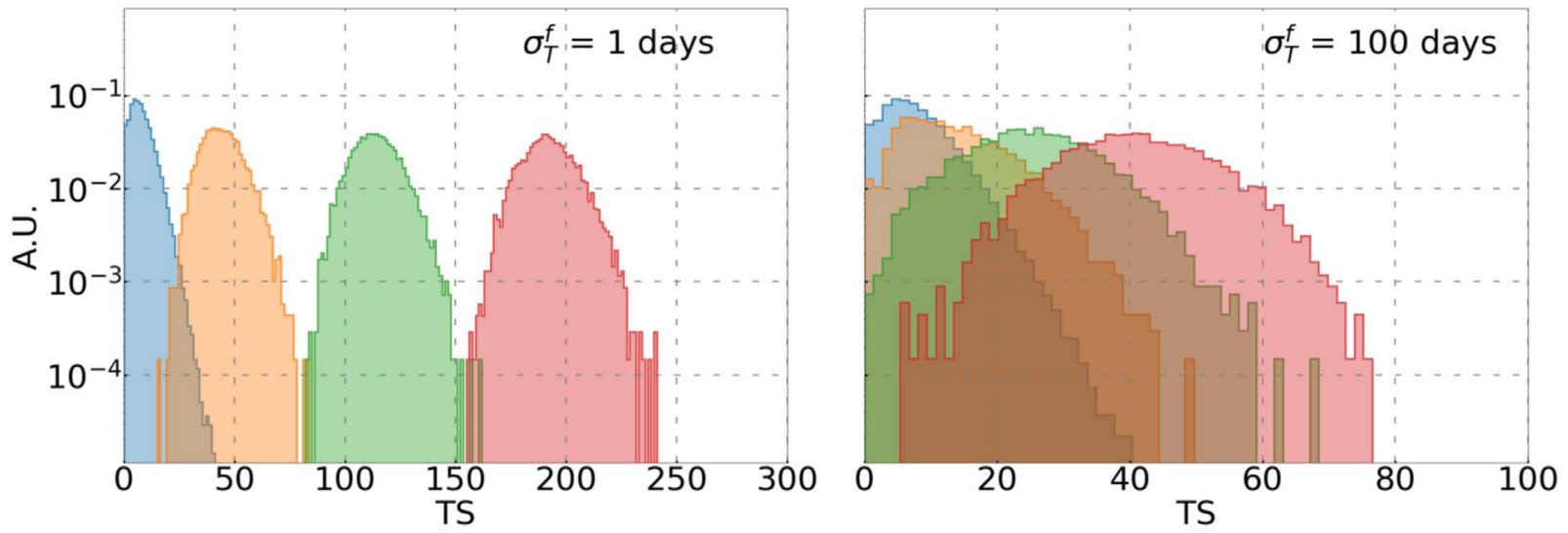

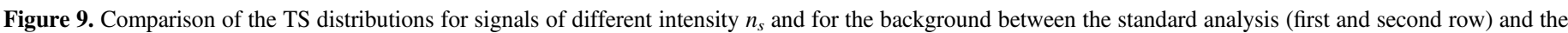

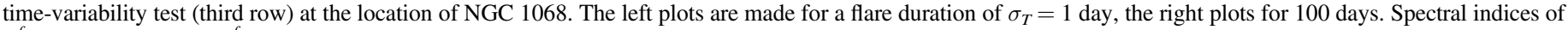
$\gamma^{f}=2$ (first row) and $\gamma^{f}=3$ (second row) are used for the injected signal in the standard analysis.

than the observed value of $3.8 \sigma$, in the assumption that the time-integrated neutrino excess is steady in time (background hypothesis). To do so, the same binomial test described in Section 3 is repeated on the list of $p$ values of the Northern sources. The per-source $p$ values are computed in the same way by comparing the TS of each source with a distribution of TS from fully scrambled (randomized times and recalculated right ascensions) background samples at the respective decl. As a matter of fact, the binomial $p$ value of the data for this test (referred to as the "posterior binomial test") is the same as reported in Section $4(3.8 \sigma)$. Nevertheless, the difference between the standard and the posterior binomial test is in the realization of the background samples used to translate the binomial $p$ value into a post-trial $p$ value.

In the posterior binomial test, background pseudo-realizations of the data for all the Northern sources of the catalog are obtained in the same way as described for the time-variability test: the times of the events are randomized while the equatorial coordinates are fixed at the recorded values such that the spatial correlations among the events are preserved and the timeintegrated information is effectively incorporated in the background hypothesis. For each pseudo-realization of the Northern catalog, the likelihood in Equation (1) is maximized at the location of each source and the corresponding TS is converted 


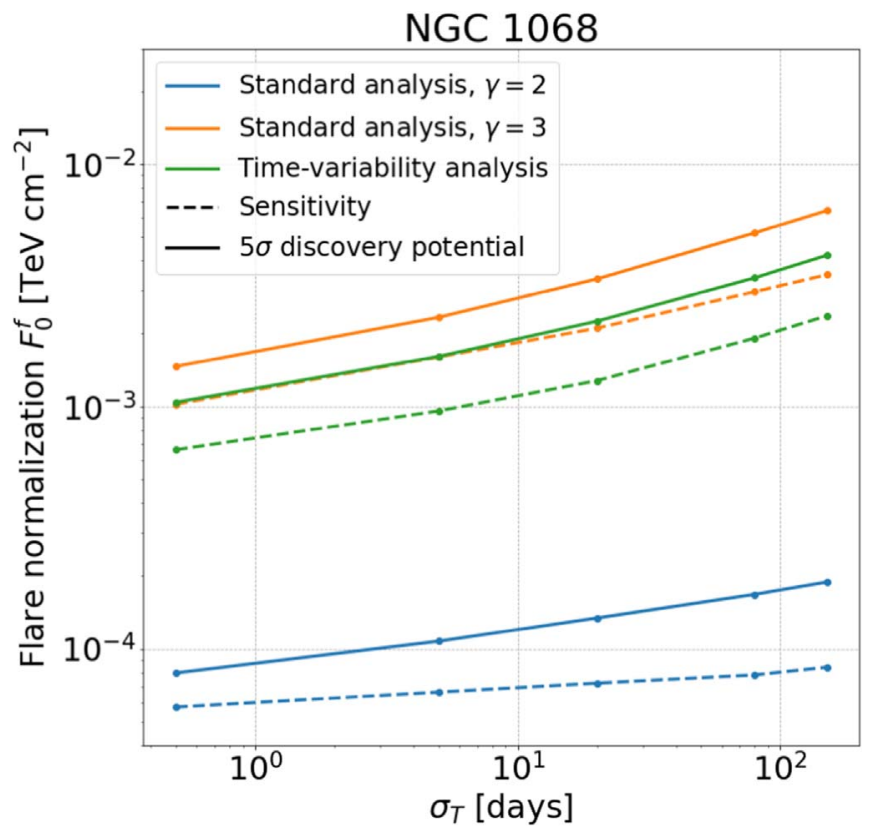

Figure 10. Comparison of the sensitivity (dashed lines) and $5 \sigma \mathrm{DP}$ (solid lines) of the standard analysis (blue and orange, respectively, for $\gamma=2$ and $\gamma=3$ ) and the time-variability test (green lines) in terms of the time-integrated flux per flare $\mathrm{F}_{0}^{f}$ described in Equation (A1). These curves are produced at the location of NGC 1068 under the hypothesis of a single signal flare. Notice that the reconstructed value of the spectral index for NGC 1068 in Aartsen et al. $(2020$ c) is 3.16 .

Table 3

Results of the Time-variability Test Applied to the Four Most Significant Sources of the Time-integrated Analysis of Aartsen et al. (2020c)

\begin{tabular}{lcc}
\hline \hline & Time-variability Results \\
\hline Source & Pre-trial $p$ Value & Post-trial $p$ Value \\
\hline NGC 1068 & 0.13 & 0.43 \\
TXS 0506+056 & 0.24 & 0.67 \\
PKS 1424+240 & 0.33 & 0.80 \\
GB6 J1542+6129 & 0.029 & 0.11 \\
\hline
\end{tabular}

Note. The table shows the $p$ values before (pre-trial) and after (post-trial) the Sidak correction with penalty factor 4 . As described in this Appendix, the timevariability test only assesses the time distribution of the recorded events, by comparing with simulated samples in which the event directions and energies remain fixed as recorded, but times are randomized according to a uniform distribution.

into a pre-trial $p$ value as described in Section 3, by comparison with a distribution of TS from fully scrambled background samples at the same decl. The lower limit on the flare duration $\sigma_{T}^{\mathrm{min}}$ is removed in this test to allow a proper comparison with the standard time-dependent binomial test. As a consequence, the azimuth-dependent correction to the background spatial PDF is neglected. However, this is a minor correction that has an impact at most of $5 \%$ only for timescales of the flares as short as $\sim 10^{-3}$ days.

Once a pre-trial $p$ value is computed for all the sources in a particular pseudo-realization of the Northern catalog, the binomial test is performed on this set of $p$ values, resulting in a background binomial $p$ value $P_{\text {bin }}$. This method is then repeated on many pseudo-realizations of the Northern catalog to produce the distribution of background binomial $p$ values for

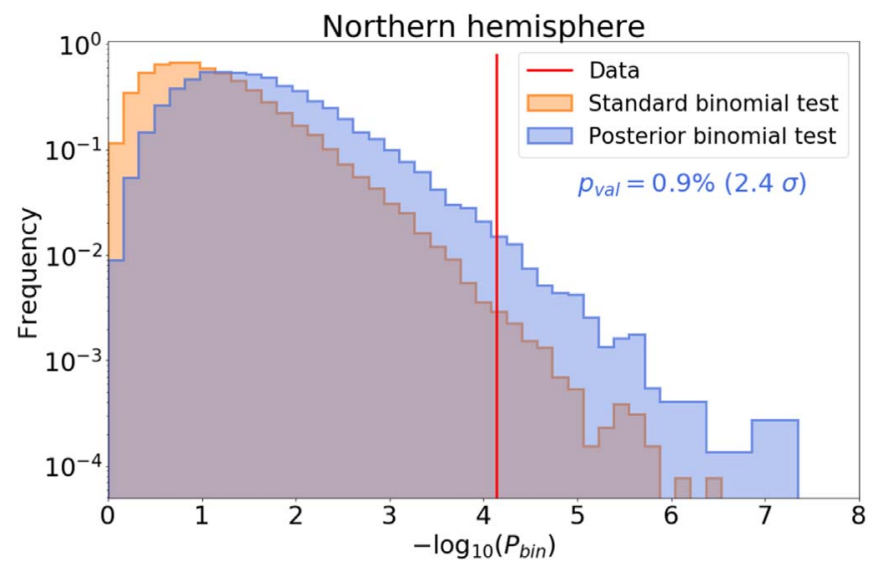

Figure 11. Background distribution of the binomial $p$ value $P_{\text {bin }}$ for the posterior (blue) and standard (orange) binomial test. For the posterior binomial test, the background sample is produced by randomizing the time of the events while keeping fixed the equatorial coordinates; for the standard analysis, the equatorial coordinates are recalculated (assuming fixed local coordinates) after the time is randomized.

the posterior binomial test shown in blue in Figure 11. These $p$ values are the typical binomial $p$ values that the binomial test produces if the neutrino events of the data (including the timeintegrated excess) have no time structure. For comparison, the orange histogram in Figure 11 is the distribution of background binomial $p$ values for the standard binomial test used in Section 4 to calculate the post-trial binomial $p$ value in the assumption that the time-integrated information is also randomized. Note that when the time-integrated information is preserved, the overall distribution is shifted toward higher values of $-\log _{10}\left(P_{\text {bin }}\right)$ as a consequence of including the additional information about the time-integrated excess in the background samples.

Finally, the probability that the time-dependent binomial test produces a more significant result than the one observed in the real data (3.8 $\sigma$ pre-trial), given the prior knowledge about the time-integrated excess and under the assumption that the neutrino events do not have any time correlation, is estimated by counting the fraction of background binomial $p$ values of the posterior binomial test that are more significant than the observed result. Such estimation leads to a probability of $0.9 \%$.

\section{Appendix D \\ Estimation of the Single-flare Significance of TXS $0506+056$}

This Appendix is intended to describe how the single-flare significances of the two flares of TXS $0506+056$, which are shown in Figure 2, are estimated.

By factorizing the background PDF, the multi-flare likelihood ratio $\Lambda_{m f}^{-1}$ in Equation (3) can be written as follows:

$$
\begin{aligned}
\Lambda_{m f}^{-1} & =\frac{\mathcal{L}\left(\hat{\boldsymbol{n}}_{s}, \hat{\gamma}, \hat{\boldsymbol{t}}_{0}, \hat{\boldsymbol{\sigma}}_{T}\right)}{\mathcal{L}\left(n_{s}=0\right)}=\prod_{j=\text { sample }} \prod_{i=1}^{N_{j}}\left(1+\sum_{f=\text { flares }} \mathcal{F}_{i, j}^{f}\right) \\
\mathcal{F}_{i, j}^{f} & :=\frac{n_{s}^{f}\left(\mathcal{S}_{i, j}^{f} / \mathcal{B}_{i, j}-1\right)}{N} .
\end{aligned}
$$

The single-flare signal and background PDFs in Equation (D.4) are the same as in Equation (2), but for the sake of clarity here they explicitly show the flare $(f)$, event $(i)$, and sample $(j)$ 
indices. In addition, the dependency on the parameters, being the same as in Equation (2), is omitted to simplify the notation.

For TXS $0506+056$ there are two identified flares, thus $\sum_{f} \mathcal{F}_{i, j}^{f}=\mathcal{F}_{i, j}^{1}+\mathcal{F}_{i, j}^{2}$. In addition, when an event $i$ does not contribute significantly to $\mathcal{F}_{i, j}^{f}$, then $\mathcal{F}_{i, j}^{f} \sim 10^{-6}-10^{-4}$. Since an event can contribute significantly only to one flare, the crossed terms $\mathcal{F}_{i, j}^{1} \mathcal{F}_{i, j}^{2}$ can be neglected and it is meaningful to retain only terms at first order in $\mathcal{F}_{i, j}^{f}$. Based on these observations, the likelihood ratio in Equation (D.4) can be well approximated as:

$$
\begin{aligned}
\Lambda_{m f}^{-1} & =\prod_{j=\text { sample }} \prod_{i=1}^{N_{j}}\left(1+\mathcal{F}_{i, j}^{1}+\mathcal{F}_{i, j}^{2}\right) \\
& \simeq \prod_{j=\text { sample }} \prod_{i=1}^{N_{j}}\left(1+\mathcal{F}_{i, j}^{1}\right)\left(1+\mathcal{F}_{i, j}^{2}\right) \\
& =\left(\Lambda_{s f}^{f=1}\right)^{-1}\left(\Lambda_{s f}^{f=2}\right)^{-1} .
\end{aligned}
$$

Thus, it can be factorized into single-flare components that are equivalent to the multi-flare likelihood ratio when only one flare is considered. This result can be easily generalized to $N_{f}>2$ flares.

Such a factorization can be exploited to disentangle the contribution of each flare to the multi-flare TS in Equation (3):

$$
\begin{aligned}
\mathrm{TS} & \simeq-2 \log \left[\frac{1}{2} \prod_{f=\text { flares }}\left(\frac{T_{\text {live }}}{\hat{\sigma}_{T}^{f} I\left[\hat{t}_{0}^{f}, \hat{\sigma}_{T}^{f}\right]}\left(\Lambda_{s f}^{f}\right)^{-1}\right)\right] \\
& =-2 \sum_{f=\text { flares }} \log \left[\left(\frac{1}{2}\right)^{1 / N_{f}} \frac{T_{\text {live }}}{\hat{\sigma}_{T}^{f} I\left[\hat{t}_{0}^{f}, \hat{\sigma}_{T}^{f}\right]}\left(\Lambda_{s f}^{f}\right)^{-1}\right] \\
& =\sum_{f=\text { flares }} \operatorname{TS}_{s f}^{f},
\end{aligned}
$$

where $\mathrm{TS}_{s f}^{f}$ is the contribution of the $f$ th flare to the multi-flare TS and can be interpreted as a single-flare TS.

The single-flare significance $\sigma_{s f}^{f}$ can be obtained in the same way as the multi-flare significance, but using the single-flare TS instead of the multi-flare TS. Assuming that the two flares of TXS $0506+056$ are independent, one might expect to retrieve the multi-flare TS by summing linearly the single-flare TS and to retrieve the multi-flare significance $\sigma_{m f}$ by summing in quadrature the single-flare significance. Although this is effectively observed for the TS, the summation in quadrature of the single-flare significance results in a mismatch of nearly $2.5 \%$ with respect to the multi-flare significance. To correct for this mismatch, the single-flare significance is redefined as $\sigma_{s f}^{\prime f}$ through the following relation:

$$
\frac{\sigma_{s f}^{\prime 1}}{\sigma_{s f}^{\prime 2}}=\frac{\sigma_{s f}^{1}}{\sigma_{s f}^{2}}, \quad \sqrt{\left(\sigma_{s f}^{\prime 1}\right)^{2}+\left(\sigma_{s f}^{\prime 2}\right)^{2}}=\sigma_{m f} .
$$

For TXS $0506+056$ this method is used to disentangle the single-flare significance $\sigma_{s f}^{\prime f}$ of the two flares used in Figure 2.

\section{Appendix E \\ Investigation of the Significance of TXS $0506+056$}

The significance of TXS $0506+056$ found by this multi-flare algorithm is smaller than the (single-flare) time-dependent significance that was determined in Aartsen et al. (2018b). The goal of this Appendix is to show that the decrease of significance is only due to the different event selection of the

\begin{tabular}{|c|c|c|}
\hline \multicolumn{3}{|c|}{ TXS 0506+056 Change in Significance } \\
\hline $\begin{array}{l}\text { Different Data Sets (multi- } \\
\text { flare, 2012-2015 only) }\end{array}$ & $\begin{array}{c}\text { PSTracks v2 } \\
\text { (Aartsen et al. 2018b) } \\
4.0 \sigma\end{array}$ & $\begin{array}{c}\text { PSTracks v3 } \\
\text { (This work) } \\
2.6 \sigma\end{array}$ \\
\hline \multirow[t]{5}{*}{$\begin{array}{r}\text { Different algorithms } \\
\text { (2012-2015 only) }\end{array}$} & $\begin{array}{l}\text { Single-flare(Aartsen } \\
\quad \text { et al. } 2018 b)\end{array}$ & $\begin{array}{l}\text { Multi-flare } \\
\text { (This work) }\end{array}$ \\
\hline & \multicolumn{2}{|c|}{ PSTracks v2 } \\
\hline & $4.0 \sigma$ & $4.0 \sigma$ \\
\hline & \multicolumn{2}{|c|}{ PSTracks v3 } \\
\hline & $2.7 \sigma$ & $2.6 \sigma$ \\
\hline \multirow{2}{*}{$\begin{array}{l}\text { Strategy of sample combina- } \\
\text { tion (single-flare, } \\
\text { PSTracks v3) }\end{array}$} & $\begin{array}{l}\text { Separate likelihoods } \\
\text { (Aartsen et al. 2018b) }\end{array}$ & $\begin{array}{c}\text { Joint likelihood } \\
\text { (This work) }\end{array}$ \\
\hline & $2.2 \sigma$ (post-trial) & $2.3 \sigma$ \\
\hline
\end{tabular}
sample used in this analysis and not due to the different likelihood algorithms. It is mainly related to two cascade events
Table 4

Results of the Comparison between the Significance Obtained for TXS 0506 +056 when using an Analysis with Features Similar to the one in Aartsen et al. (2018b) and the One Presented in This Paper

Note. When testing the impact of different data sets, the years 2012-2015 are analyzed with the multi-flare algorithm. When testing the impact of a different strategy in the combination of the samples, the single-flare algorithm is used on the data set PSTracks v3. In one case only, the IceCube sample containing the known flare is analyzed and the $p$ value penalized, adopting the same logic as in Aartsen et al. (2018b); in the other case, all the $10 \mathrm{yr}$ samples are combined in a joint likelihood, as described in Section 3, and no penalization is needed.

that are rejected in the new event selection, presented in Aartsen et al. (2020c). This was discussed also in IceCube (Abbasi et al. 2021b). As a matter of fact, the new selection was focused on muon tracks for achieving best angular resolutions for the point-source search.

The differences between this analysis and the one described in Aartsen et al. (2018b) are mainly of three types. These are investigated using the analysis described in this Letter and the one presented in Aartsen et al. (2018b) to find out how much each of them contributes to the change in significance of TXS $0506+056$. The results are summarized in Table 4 .

Different data sets: As also mentioned in Section 2, the event selections used to produce the data set analyzed in Aartsen et al. (2018b) and the one analyzed in this work (from Aartsen et al. 2020c) are different. According to the internal IceCube nomenclature, the two data sets are referred to as PSTracks $\mathrm{V} 2$ and PSTracks V3, respectively. In some cases, the different event selection results in the reconstruction of slightly different energy and local angles. An extensive and detailed description of the two data sets can be found in Abbasi et al. (2021b).

The significance of TXS 0506+056 is estimated on PSTracks v2 and PSTracks v3 by applying the multiflare algorithm to the years 2012-2015 (containing only one of the two flares detected by this analysis). We observe the same drop in significance (from $4.0 \sigma$ in PSTracks $v 2$ to $2.6 \sigma$ in PSTracks v3) described in Abbasi et al. (2021b). The significance observed for PSTracks $\mathrm{V} 3$ increases to $3.4 \sigma$ if the two high-energy events, present in PSTracks V2 but absent in PSTracks v3, are added by hand to the data set. It is also worth noting that the pre-trial significance observed for PSTracks V2 with the multi-flare algorithm is not different from the pre-trial significance reported in Aartsen et al. (2018b), which was obtained with a single-flare algorithm. 
Different algorithms: The multi-flare algorithm has been developed for this analysis and applied for the first time in this work. This is a crucial difference between this work and the one presented in Aartsen et al. (2018b), since a multi-flare likelihood could in principle consist of more fit parameters than a single-flare likelihood. The increased parameter space of the fit may thus degrade the sensitivity. This degradation was avoided by requiring a pre-selection of candidate flares with $\mathrm{TS} \geqslant 2$ (see Section 3 and Appendix B). Other minor improvements between the two analyses concern a Gaussian integral factor included in the marginalization term to correct for boundary effects, the time PDF normalization, set to 1 across each IceCube sample by considering only up times of the detector (in Aartsen et al. [2018b] it was set to 1 in an infinite range, regardless of the up times). The results, shown in Table 4 for the single- and multi-flare algorithm applied to the 2012-2015 data, suggest that the multi-flare algorithm is not responsible for the drop of the significance, when applied to the same data set.

Different strategies for combining independent samples: The third and last potential source of change in significance is due to the different strategies adopted to combine the IceCube samples. Since the $10 \mathrm{yr}$ data sample of IceCube concerns different IceCube detector configurations, triggers, and event cuts, this analysis is based on the maximization of the joint likelihood defined as the product of the likelihoods of each IceCube sample (see Section 3). The strategy adopted in Aartsen et al. (2018b) instead consists of maximizing the likelihood of each IceCube sample, picking up the most significant $p$ value and reporting it as post-trial after correcting for the look-elsewhere effect. Such a correction is made by penalizing the most significant $p$ value by the ratio of the live time of the sample with the most significant $p$ value to the total time. To investigate this difference, the single-flare algorithm is applied to PSTracks V3. To reproduce the analysis in (Aartsen et al. 2018b), the TS is maximized only across the $3 \mathrm{yr}$ between 2012-2015 (containing the most significant flare) and the $p$ value is penalized by the ratio of 10-3 yr, adopting the same logic described in Aartsen et al. (2018b). In the analysis presented in this Letter, the whole $10 \mathrm{yr}$ data are analyzed with a single joint likelihood (as described in Section 3 but without the multiple flare feature) and the same penalization of the $p$ value is not needed in this case. As seen in Table 4, it can be stated that the results obtained in the two cases are comparable and that the strategy adopted to combine the different samples is not responsible for a substantial change in significance.

\section{Appendix F \\ Discussion on the Multi-messenger Context}

As shown in Section 4, M87 is the most significant source of the catalog, exhibiting three events over a time lag of minute scale with post-trail significance of $1.7 \sigma$. It is one of the closest $(z=0.00436)$ potential $\mathrm{CR}$ accelerators, hosting a supermassive black hole of $6.5 \times 10^{9} \mathrm{M}_{\odot}$. Its jet was already observed more than a century ago (Blandford et al. 2019) in a large elliptical radio galaxy of Fanaroff-Riley type I in the Virgo cluster. It has been observed in $>100 \mathrm{GeV}$ energy region: VERITAS detected a flare extending beyond $350 \mathrm{GeV}$ with a spectral index at the peak of $2.19 \pm 0.07$ (Aliu et al. 2012) in 2010 April. In a 2008 flare, a clear correlation between the $\mathrm{X}$-ray emission and the $\mathrm{TeV}$ one was noted by Acciari et al. (2008) and Albert et al. (2008). A previous positive detection was reported by HEGRA in 1998/99 above $700 \mathrm{GeV}$ (Aharonian et al. 2003), and up to $\sim 10 \mathrm{TeV}$ by H.E.S.S. in 89 hours of observation between 2003-2006, showing a variability at the timescale of a few days in the 2005 high state associated with the Schwarzschild radius of M87 Aharonian et al. (2006). Recently, MAGIC reported the results on the monitoring of M87 for 156 hours in 2012-15 Acciari et al. (2020). It is worth noting that HAWC set an upper limit above $2 \mathrm{TeV}$ for 760 days of data. The non-observation of gamma-rays at $>\mathrm{TeV}$ energies may indicate a cut-off in the spectrum. Such a cut-off may differ for neutrinos, being less affected by the absorption in the source and by the extragalactic background light.

The gamma-ray observations from M87 are summarized in Figure 12, together with the $10 \mathrm{yr}$ time-integrated upper limits on the neutrino flux estimated in Aartsen et al. (2020c) for a spectrum of the form $d N / d E \sim E^{-2}$.

Precise radio observations (Sikora et al. 2016) indicate a persistent central ridge structure, namely a spine flow in the interior of M87 jet, in addition to the well-known limbbrightening profile, which needs further measurements. A composite structure of the jet has been speculated also for TXS $0506+056$ based on observations months after the detection of the IceCube high-energy event that triggered its multiwavelength observations. With the millimeter-VLBI it was observed that the core jet expands in size with apparent superluminal velocity (Ros et al. 2020). This can be interpreted as deceleration due to proton loading from jet-star interactions in the inner host galaxy and/or spine-sheath structure of the jet (Ghisellini et al. 2005; Tavecchio \& Ghisellini 2008). This sort of spine-sheat structure has been advocated as a possible explanation for the higher flux of neutrinos than gamma-rays and also suggested for TXS 0506+056 by MAGIC (Ansoldi et al. 2018), while models with a single zone struggle to explain the 2014-2015 flare of TXS 0506+056 (see, e.g., Keivani et al. 2018; Murase et al. 2018; Zhang et al. 2020).

Other models (e.g., Inoue et al. 2020; Murase et al. 2020), have been revised to explain the more recent observations of IceCube on NGC 1068 (Aartsen et al. 2020c). These models focus on the higher observed flux of IceCube neutrino events in the $\sim 1-50 \mathrm{TeV}$ region with respect to the level of gamma-ray fluxes observed at lower energy by Fermi and the limits of MAGIC. The corona super-hot plasma around the supermassive black hole accelerates protons, carrying a few percent of the thermal energy through plasma turbulence (Murase et al. 2020) or shock acceleration (Inoue et al. 2020), leading to the creation of neutrinos and gamma-rays. The environment is dense enough to prevent the escape of $\gg 100 \mathrm{MeV}$ gamma-rays while $\sim \mathrm{MeV}$ gamma-rays would be their result from cascading down. Further insights will be needed in both messengers and all wavelengths to better constrain the structure of jets and acceleration mechanisms in one or multiple zones. 


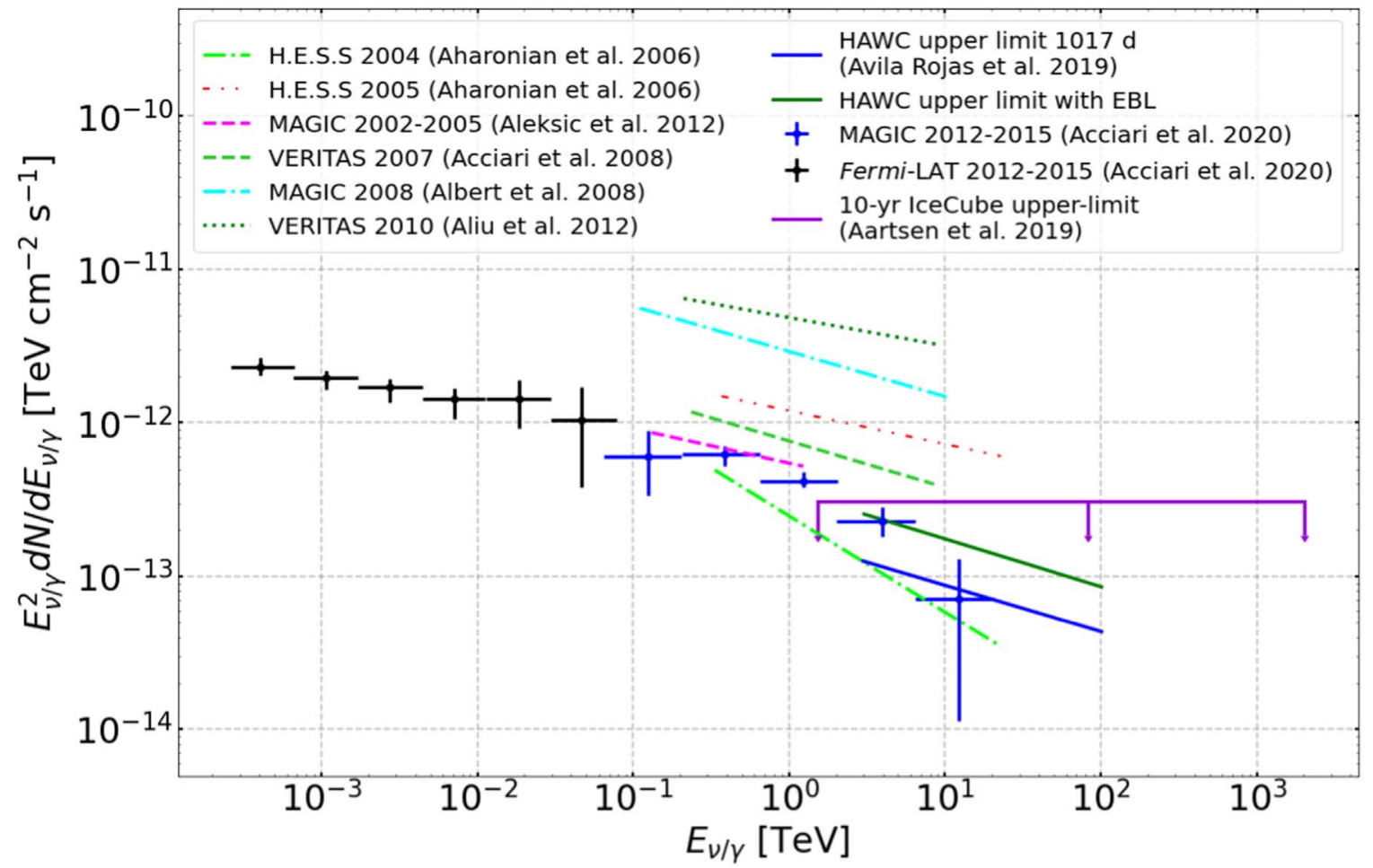

Figure 12. The gamma-ray flux in the steady state of the source observed between 2012-2015 (Acciari et al. 2020) is shown with black (Fermi-LAT) and blue dots (MAGIC). The higher-level dashed lines are levels of flux observed during flares (see references in the text). The purple line with downing arrows corresponds to the $10 \mathrm{yr}$ time-integrated upper limits taken from Aartsen et al. (2020c), with an assumed spectrum $d N / d E \sim E^{-2}$.

\section{ORCID iDs}

R. Abbasi (i) https://orcid.org/0000-0001-6141-4205 M. Ackermann (ii) https://orcid.org/0000-0001-8952-588X J. A. Aguilar (1) https://orcid.org/0000-0003-2252-9514 M. Ahlers (ib https://orcid.org/0000-0003-0709-5631 G. Anton (1) https://orcid.org/0000-0003-2039-4724 C. Argüelles (1) https://orcid.org/0000-0003-4186-4182 A. Balagopal V. (10 https://orcid.org/0000-0001-5367-8876

A. Barbano iㅡ https://orcid.org/0000-0002-4836-7093

S. W. Barwick (10 https://orcid.org/0000-0003-2050-6714

V. Basu (1) https://orcid.org/0000-0002-9528-2009

S. Baur (1) https://orcid.org/0000-0002-3329-1276

J. J. Beatty (1) https://orcid.org/0000-0003-0481-4952

J. Becker Tjus (1) https://orcid.org/0000-0002-1748-7367

C. Bellenghi (1) https://orcid.org/0000-0001-8525-7515

S. BenZvi 지 https://orcid.org/0000-0001-5537-4710

E. Bernardini (1) https://orcid.org/0000-0003-3108-1141

D. Z. Besson (1) https://orcid.org/0000-0001-6733-963X

E. Blaufuss (1) https://orcid.org/0000-0001-5450-1757

S. Blot (i) https://orcid.org/0000-0003-1089-3001

S. Böser (1) https://orcid.org/0000-0002-5918-4890

O. Botner (ㄷ) https://orcid.org/0000-0001-8588-7306

F. Bradascio (1) https://orcid.org/0000-0002-7750-5256

A. Burgman (1) https://orcid.org/0000-0003-1276-676X

M. A. Campana (i) https://orcid.org/0000-0003-4162-5739

C. Chen (i) https://orcid.org/0000-0002-2813-7688

D. Chirkin (1) https://orcid.org/0000-0003-4911-1345

B. A. Clark (ib https://orcid.org/0000-0003-4089-2245

K. Clark (1) https://orcid.org/0000-0003-2467-6825

A. Coleman (1) https://orcid.org/0000-0003-1510-1712

J. M. Conrad (1) https://orcid.org/0000-0002-6393-0438

P. Coppin (1) https://orcid.org/0000-0001-6869-1280
P. Correa (1) https://orcid.org/0000-0002-1158-6735

D. F. Cowen (1) https://orcid.org/0000-0003-4738-0787

R. Cross (16) https://orcid.org/0000-0003-0081-8024

C. Dappen (i) https://orcid.org/0000-0002-4188-9219

P. Dave (i) https://orcid.org/0000-0002-3879-5115

C. De Clercq (i) https://orcid.org/0000-0001-5266-7059

J. J. DeLaunay (1) https://orcid.org/0000-0001-5229-1995

H. Dembinski (1) https://orcid.org/0000-0003-3337-3850

A. Desai (i) https://orcid.org/0000-0001-7405-9994

P. Desiati $\odot$ https://orcid.org/0000-0001-9768-1858

K. D. de Vries (ํ) https://orcid.org/0000-0002-9842-4068

G. de Wasseige (1) https://orcid.org/0000-0002-1010-5100

T. DeYoung (1) https://orcid.org/0000-0003-4873-3783

A. Diaz (1) https://orcid.org/0000-0001-7206-8336

J. C. Díaz-Vélez (i) https://orcid.org/0000-0002-0087-0693

H. Dujmovic (다 https://orcid.org/0000-0003-1891-0718

M. A. DuVernois (16) https://orcid.org/0000-0002-2987-9691

P. Eller (1) https://orcid.org/0000-0001-6354-5209

P. A. Evenson (1) https://orcid.org/0000-0001-7929-810X

K. L. Fan (i) https://orcid.org/0000-0002-8246-4751

A. R. Fazely (1) https://orcid.org/0000-0002-6907-8020

A. T. Fienberg (1) https://orcid.org/0000-0002-9472-3597

C. Finley (1) https://orcid.org/0000-0003-3350-390X

D. Fox (i) https://orcid.org/0000-0002-3714-672X

A. Franckowiak (i) https://orcid.org/0000-0002-5605-2219

T. K. Gaisser (1) https://orcid.org/0000-0003-4717-6620

E. Ganster (1) https://orcid.org/0000-0003-4393-6944

A. Garcia (ㄷ) https://orcid.org/0000-0002-8186-2459

S. Garrappa (1) https://orcid.org/0000-0003-2403-4582

A. Ghadimi (1) https://orcid.org/0000-0002-6350-6485

C. Glaser (1) https://orcid.org/0000-0001-5998-2553

T. Glauch (1) https://orcid.org/0000-0003-1804-4055

T. Glüsenkamp (1) https://orcid.org/0000-0002-2268-9297 
S. Goswami (1) https://orcid.org/0000-0002-0373-9770

S. Griswold (10) https://orcid.org/0000-0002-7321-7513

A. Hallgren (i) https://orcid.org/0000-0001-7751-4489

L. Halve (i) https://orcid.org/0000-0003-2237-6714

F. Halzen (1) https://orcid.org/0000-0001-6224-2417

A. Haungs (i) https://orcid.org/0000-0002-9638-7574

K. Helbing (1) https://orcid.org/0000-0003-2072-4172

F. Henningsen (1) https://orcid.org/0000-0002-0680-6588

C. Hill (i) https://orcid.org/0000-0003-0647-9174

F. Huang (i) https://orcid.org/0000-0002-6014-5928

T. Huber (i) https://orcid.org/0000-0002-6515-1673

N. Iovine (i) https://orcid.org/0000-0001-7965-2252

G. S. Japaridze (1) https://orcid.org/0000-0002-7000-5291

B. J. P. Jones (i) https://orcid.org/0000-0003-3400-8986

D. Kang (1) https://orcid.org/0000-0002-5149-9767

W. Kang (1) https://orcid.org/0000-0003-3980-3778

A. Kappes (ㄴ) https://orcid.org/0000-0003-1315-3711

T. Karg (1) https://orcid.org/0000-0003-3251-2126

M. Karl (i) https://orcid.org/0000-0003-2475-8951

A. Karle (i) https://orcid.org/0000-0001-9889-5161

U. Katz (i) https://orcid.org/0000-0002-7063-4418

M. Kauer (1) https://orcid.org/0000-0003-1830-9076

J. L. Kelley (1) https://orcid.org/0000-0002-0846-4542

A. Kheirandish (1) https://orcid.org/0000-0001-7074-0539

S. R. Klein (1) https://orcid.org/0000-0003-2841-6553

R. Koirala @ https://orcid.org/0000-0002-7735-7169

H. Kolanoski (i) https://orcid.org/0000-0003-0435-2524

L. Köpke (i) https://orcid.org/0000-0001-8530-6348

C. Kopper (i) https://orcid.org/0000-0001-6288-7637

D. J. Koskinen (1) https://orcid.org/0000-0002-0514-5917

P. Koundal iㅣ https://orcid.org/0000-0002-5917-5230

M. Kovacevich (1) https://orcid.org/0000-0002-5019-5745

M. Kowalski (i) https://orcid.org/0000-0001-8594-8666

N. Kurahashi (10) https://orcid.org/0000-0003-1047-8094

C. Lagunas Gualda (1) https://orcid.org/0000-0002-9040-7191

M. J. Larson (1) https://orcid.org/0000-0002-6996-1155

F. Lauber (1) https://orcid.org/0000-0001-5648-5930

J. P. Lazar (1) https://orcid.org/0000-0003-0928-5025

K. Leonard (1) https://orcid.org/0000-0002-8795-0601

A. Leszczyńska (i) https://orcid.org/0000-0003-0935-6313

Q. R. Liu (1) https://orcid.org/0000-0003-3379-6423

E. Lohfink (1) https://orcid.org/0000-0003-3248-5682

L. Lu (1) https://orcid.org/0000-0003-3175-7770

F. Lucarelli (1) https://orcid.org/0000-0002-9558-8788

A. Ludwig (1) https://orcid.org/0000-0001-9038-4375

W. Luszczak (1) https://orcid.org/0000-0003-3085-0674

Y. Lyu (i) https://orcid.org/0000-0002-2333-4383

W. Y. Ma (1) https://orcid.org/0000-0003-1251-5493

J. Madsen $\odot$ https://orcid.org/0000-0003-2415-9959

I. C. Mariş (ํ) https://orcid.org/0000-0002-5771-1124

R. Maruyama (i) https://orcid.org/0000-0003-2794-512X

F. McNally (i) https://orcid.org/0000-0002-0785-2244

K. Meagher (i) https://orcid.org/0000-0003-3967-1533

M. Meier (1) https://orcid.org/0000-0002-9483-9450

S. Meighen-Berger (1) https://orcid.org/0000-0001-6579-2000

T. Montaruli (1) https://orcid.org/0000-0001-5014-2152

R. W. Moore (1) https://orcid.org/0000-0003-4160-4700

M. Moulai 주 https://orcid.org/0000-0001-7909-5812

R. Naab (ㄷ) https://orcid.org/0000-0003-2512-466X

R. Nagai (1) https://orcid.org/0000-0001-7503-2777

J. Necker (1) https://orcid.org/0000-0003-0280-7484

H. Niederhausen (1) https://orcid.org/0000-0002-9566-4904
M. U. Nisa (1) https://orcid.org/0000-0002-6859-3944

A. Obertacke Pollmann (i) https://orcid.org/0000-00022492-043X

B. Oeyen (1) https://orcid.org/0000-0003-2940-3164

E. O'Sullivan (1) https://orcid.org/0000-0003-1882-8802

H. Pandya (i) https://orcid.org/0000-0002-6138-4808

N. Park (1) https://orcid.org/0000-0002-4282-736X

E. N. Paudel (1) https://orcid.org/0000-0001-9276-7994

C. Pérez de los Heros (ib https://orcid.org/0000-0002-2084-5866

J. Peterson (1) https://orcid.org/0000-0002-7985-1443

A. Pizzuto (i) https://orcid.org/0000-0002-8466-8168

M. Plum (16 https://orcid.org/0000-0001-8691-242X

A. Porcelli (i) https://orcid.org/0000-0002-3220-6295

C. Raab (i) https://orcid.org/0000-0001-9921-2668

M. Rameez (i) https://orcid.org/0000-0001-5023-5631

A. Rehman (1) https://orcid.org/0000-0001-7616-5790

R. Reimann (1) https://orcid.org/0000-0002-1983-8271

E. Resconi (1) https://orcid.org/0000-0003-0705-2770

S. Reusch 다 https://orcid.org/0000-0002-7788-628X

W. Rhode (1) https://orcid.org/0000-0003-2636-5000

B. Riedel (i) https://orcid.org/0000-0002-9524-8943

M. Rongen (iD https://orcid.org/0000-0002-7057-1007

C. Rott (1) https://orcid.org/0000-0002-6958-6033

D. Ryckbosch (1) https://orcid.org/0000-0002-8759-7553

D. Rysewyk Cantu (1) https://orcid.org/0000-0002-3612-6129

I. Safa (1) https://orcid.org/0000-0001-8737-6825

A. Sandrock (1) https://orcid.org/0000-0002-6779-1172

J. Sandroos (i) https://orcid.org/0000-0002-0629-0630

M. Santander (1) https://orcid.org/0000-0001-7297-8217

S. Sarkar (1) https://orcid.org/0000-0002-3542-858X

S. Sarkar (i) https://orcid.org/0000-0002-1206-4330

K. Satalecka (1) https://orcid.org/0000-0002-7669-266X

A. Schneider (1) https://orcid.org/0000-0002-0895-3477

J. Schneider (1) https://orcid.org/0000-0001-7752-5700

F. G. Schröder (i) https://orcid.org/0000-0001-8495-7210

L. Schumacher (10 https://orcid.org/0000-0001-8945-6722

S. Sclafani (1) https://orcid.org/0000-0001-9446-1219

A. Sharma (1) https://orcid.org/0000-0001-5397-6777

M. Silva (i) https://orcid.org/0000-0001-6940-8184

B. Smithers (1) https://orcid.org/0000-0003-1273-985X

J. Soedingrekso (1) https://orcid.org/0000-0003-1011-2797

D. Soldin (1) https://orcid.org/0000-0003-3005-7879

G. M. Spiczak (ㄷ) https://orcid.org/0000-0002-0030-0519

C. Spiering (i) https://orcid.org/0000-0001-7372-0074

J. Stachurska (i) https://orcid.org/0000-0002-0238-5608

R. Stein (1) https://orcid.org/0000-0003-2434-0387

J. Stettner 자 https://orcid.org/0000-0003-1042-3675

T. Stezelberger (1) https://orcid.org/0000-0003-2676-9574

T. Stuttard (1) https://orcid.org/0000-0001-7944-279X

G. W. Sullivan (®i) https://orcid.org/0000-0002-2585-2352

I. Taboada (1) https://orcid.org/0000-0003-3509-3457

F. Tenholt (i) https://orcid.org/0000-0002-7156-7392

S. Ter-Antonyan (1) https://orcid.org/0000-0002-5788-1369

K. Tollefson (1) https://orcid.org/0000-0001-9725-1479

L. Tomankova (1) https://orcid.org/0000-0003-0696-7119

S. Toscano (ib https://orcid.org/0000-0002-1860-2240

A. Trettin (ㄷ) https://orcid.org/0000-0003-0350-3597

C. F. Tung (1) https://orcid.org/0000-0001-6920-7841

A. Turcati (i) https://orcid.org/0000-0002-8050-7869

C. F. Turley (1) https://orcid.org/0000-0002-9689-8075

M. A. Unland Elorrieta $(1)$ https://orcid.org/0000-00026124-3255 
J. Vandenbroucke (ib https://orcid.org/0000-0002-9867-6548

N. van Eijndhoven (1) https://orcid.org/0000-0001-5558-3328

J. van Santen (ib https://orcid.org/0000-0002-2412-9728

S. Verpoest (ib https://orcid.org/0000-0002-3031-3206

T. B. Watson (1) https://orcid.org/0000-0002-8631-2253

C. Weaver (1) https://orcid.org/0000-0003-2385-2559

J. Weldert (1) https://orcid.org/0000-0002-3709-2354

C. Wendt (1) https://orcid.org/0000-0001-8076-8877

N. Whitehorn (10 https://orcid.org/0000-0002-3157-0407

C. H. Wiebusch $\mathbb{1}$ (i) https://orcid.org/0000-0002-6418-3008

M. Wolf (1) https://orcid.org/0000-0001-9991-3923

S. Yoshida (1) https://orcid.org/0000-0003-2480-5105

T. Yuan (1) https://orcid.org/0000-0002-7041-5872

Z. Zhang (1) https://orcid.org/0000-0002-7347-283X

\section{References}

Aartsen, M. G., Abbasi, R., Abdou, Y., IceCube, et al. 2013a, Sci, 342, 1242856

Aartsen, M. G., Abbasi, R., Abdou, Y., et al. 2013b, ApJ, 779, 132

Aartsen, M. G., Abbasi, R., Abdou, Y., et al. 2017a, ApJ, 835, 45

Aartsen, M. G., Abbasi, R., Abdou, Y., et al. 2017b, ApJ, 835, 151

Aartsen, M. G., Abraham, K., Ackermann, M., et al. 2016, ApJ, 833, 3

Aartsen, M. G., Ackermann, M., Adams, J., et al. 2014a, PhRvL, 113, 101101

Aartsen, M. G., Ackermann, M., Adams, J., et al. 2014b, ApJ, 796, 109

Aartsen, M. G., Ackermann, M., Adams, J., et al. 2015, ApJ, 807, 46

Aartsen, M. G., Ackermann, M., Adams, J., et al. 2017c, arXiv:1710.01191

Aartsen, M. G., Ackermann, M., Adams, J., et al. 2017d, ApJ, 843, 112

Aartsen, M. G., Ackermann, M., Adams, J., et al. 2018a, Sci, 361, eaat1378

Aartsen, M. G., Ackermann, M., Adams, J., et al. 2018b, Sci, 361, 147

Aartsen, M. G., Ackermann, M., Adams, J., et al. 2020a, ApJL, 898, L10

Aartsen, M. G., Ackermann, M., Adams, J., et al. 2020b, ApJ, 898, 117

Aartsen, M. G., Ackermann, M., Adams, J., et al. 2020c, PhRvL, 124, 051103

Abbasi, R., Abdou, Y., Abu-Zayyad, T., et al. 2010, NIMPA, 618, 139

Abbasi, R., Abdou, Y., Abu-Zayyad, T., et al. 2011, ApJ, 732, 18

Abbasi, R., Ackermann, M., Adams, J., et al. 2009, NIMPA, 601, 294

Abbasi, R., Ackermann, M., Adams, J., et al. 2021a, ApJ, 911, 67

Abbasi, R., Ackermann, M., Adams, J., et al. 2021b, arXiv:2101.09836
Abdi, H. 2007, in Encyclopedia of Measurement and Statistics, ed. N. J. Salkind (Thousand Oaks, CA: Sage), 103

Abdollahi, S., Acero, F., Ackermann, M., et al. 2020, ApJS, 247, 33

Acciari, V. A., Ansoldi, S., Antonelli, L. A., et al. 2020, MNRAS, 492, 5354

Acciari, V. A., Beilicke, M., Blaylock, G., et al. 2008, ApJ, 679, 397

Achterberg, A., Ackermann, M., Adams, J., et al. 2006, APh, 26, 155

Aharonian, F., Akhperjanian, A., Beilicke, M., et al. 2003, A\&A, 403, L1

Aharonian, F., Akhperjanian, A. G., Bazer-Bachi, A. R., et al. 2006, Sci, 314,1424

Albert, J., Aliu, E., Anderhub, H., et al. 2008, ApJL, 685, L23

Aliu, E., Arlen, T., Aune, T., et al. 2012, ApJ, 746, 141

Ansoldi, S., Antonelli, L. A., Arcaro, C., et al. 2018, ApJL, 863, L10

Blandford, R., Meier, D., \& Readhead, A. 2019, ARA\&A, 57, 467

Braun, J., Baker, M., Dumm, J., et al. 2010, APh, 33, 175

Desiati, P., Jagielski, K., Schukraft, A., et al. 2013, in 33rd ICRC, Seasonal variation of atmospheric neutrinos in IceCube, ed. A. Saa, http://adsabs. harvard.edu/pdf/2013ICRC...33.3240D

gammaCat. 2018, GammaCat: online catalogue of Gamma-ray sources., https://gamma-cat.readthedocs.io/data/overview.html

Garrappa, S., Buson, S., Franckowiak, A., et al. 2019, ApJ, 880, 103

Ghisellini, G., Tavecchio, F., \& Chiaberge, M. 2005, A\&A, 432, 401

Inoue, Y., Khangulyan, D., \& Doi, A. 2020, ApJL, 891, L33

Keivani, A., Murase, K., Petropoulou, M., et al. 2018, ApJ, 864, 84

Murase, K., Kimura, S. S., \& Mészáros, E. 2020, PhRvL, 011101, 125

Murase, K., Oikonomou, F., \& Petropoulou, M. 2018, ApJ, 865, 124

O'Sullivan, E., \& Finley, C. 2019, in 36th ICRC, Searching for TimeDependent Neutrino Emission from Blazars with IceCube, 358, 973, https://pos.sissa.it/358/973/

Padovani, P., Giommi, P., Glauce, T., et al. 2018, MNRAS, 480, 192

Padovani, P., Oikonomou, F., Petropoulou, M., Giommi, P., \& Resconi, E. 2019, MNRAS, 484, L104

Ros, E., Kadler, M., Perucho, M., et al. 2020, A\&A, 633, L1

Sikora, M., Rutkowski, M., \& Begelman, M. C. 2016, MNRAS, 457, 1352

Stein, R. 2020, in 36th International Cosmic Ray Conference, Search for Neutrinos from Populations of Optical Transients, 358, 1016, https://pos. sissa.it/358/1016

Tavecchio, F., \& Ghisellini, G. 2008, MNRAS, 385, 98

Wakely, S. P., \& Horan, D. 2008, in 30th ICRC, TeVCat: An online catalog for Very High Energy Gamma-Ray Astronomy, 3, 1341

Zhang, B. T., Petropoulou, M., Murase, K., \& Oikonomou, F. 2020, ApJ, 889,118 